Supporting Information

for

\title{
A Promiscuous Proton in Taxadiene Biosynthesis?
}

\author{
Pradeep Gutta and Dean J. Tantillo* \\ Department of Chemistry \\ University of California, Davis \\ One Shields Avenue \\ Davis, CA 95616
}

tantillo@chem.ucdavis.edu 
All calculations were performed with Gaussian03 (M. J. Frisch, et al., Gaussian, Inc., Pittsburgh $P A, 2003$, full reference below). Geometries were optimized using the B3LYP/6-31+G(d,p) method (Becke, A. D. J. Chem. Phys. 1993, 98, 5648-5652; Becke, A. D. J. Chem. Phys. 1993, 98, 1372-1377; Lee, C.; Yang, W.; Parr, R. G. Phys. Rev. B 1988, 37, 785-789; Stephens, P. J.; Devlin, F. J.; Chabalowski, C. F.; Frisch, M. J. J. Phys. Chem. 1994, 98, 11623-11627). All structures were characterized by frequency calculations, and reported energies include zero-point energy corrections (unscaled). Intrinsic reaction coordinate (IRC) calculations (Gonzalez, C.; Schlegel, H. B. J. Phys. Chem. 1990, 94, 5523-5527; Fukui, K. Acc. Chem. Res. 1981, 14, 363368) were also used to characterize transition structures. Structural drawings were produced using Ball \& Stick (N. Müller and A. Falk, Ball \& Stick V.3.7.6, molecular graphics application for MacOS computers, Johannes Kepler University Linz, 2000).

\section{Gaussian 03, Revision B.04,}

M. J. Frisch, G. W. Trucks, H. B. Schlegel, G. E. Scuseria,

M. A. Robb, J. R. Cheeseman, J. A. Montgomery, Jr., T. Vreven,

K. N. Kudin, J. C. Burant, J. M. Millam, S. S. Iyengar, J. Tomasi,

V. Barone, B. Mennucci, M. Cossi, G. Scalmani, N. Rega,

G. A. Petersson, H. Nakatsuji, M. Hada, M. Ehara, K. Toyota,

R. Fukuda, J. Hasegawa, M. Ishida, T. Nakajima, Y. Honda, O. Kitao,

H. Nakai, M. Klene, X. Li, J. E. Knox, H. P. Hratchian, J. B. Cross,

C. Adamo, J. Jaramillo, R. Gomperts, R. E. Stratmann, O. Yazyev,

A. J. Austin, R. Cammi, C. Pomelli, J. W. Ochterski, P. Y. Ayala,

K. Morokuma, G. A. Voth, P. Salvador, J. J. Dannenberg,

V. G. Zakrzewski, S. Dapprich, A. D. Daniels, M. C. Strain,

O. Farkas, D. K. Malick, A. D. Rabuck, K. Raghavachari,

J. B. Foresman, J. V. Ortiz, Q. Cui, A. G. Baboul, S. Clifford,

J. Cioslowski, B. B. Stefanov, G. Liu, A. Liashenko, P. Piskorz,

I. Komaromi, R. L. Martin, D. J. Fox, T. Keith, M. A. Al-Laham,

C. Y. Peng, A. Nanayakkara, M. Challacombe, P. M. W. Gill,

B. Johnson, W. Chen, M. W. Wong, C. Gonzalez, and J. A. Pople,

Gaussian, Inc., Pittsburgh PA, 2003.

Note that a recent report suggested that B3LYP may systematically underestimate the reaction energies for carbocation-alkene cyclization reactions and therefore suggested that mPW1PW91 single point calculations may improve the energetics in such systems (Matsuda, S. P. T.; Wilson, W. K.; Xiong, Q. Org. Biomol. Chem. 2006, 4, 530-543). For comparison, the relative energies of 3, 4, 6, TS 3-to-4, $\mathbf{T S}_{\text {4-to-6, }}$ and $\mathbf{T S}_{\text {3-to-6 }}$ at the mPW1PW91/6-31+G(d,p)//B3LYP/6-31+G(d,p) level (without zero point energy corrections) are, respectively: [0.00]. $+2.20,+0.31,+11.63,+5.84$, and +5.47 . Note that these values are all very close to those computed with $B 3 L Y P / 6-31+G(d, p)+$ $\mathrm{ZPE}(\mathrm{B} 3 \mathrm{LYP} / 6-31+\mathrm{G}(\mathrm{d}, \mathrm{p}))$ and reported in the text. 
Structure 3:

No imaginary frequencies

$\mathrm{E}(\mathrm{b} 3$ lyp $)=-781.76222$ A.U.

Zero-point correction $=0.48485$ (Hartree/Particle)

Sum of electronic and zero-point Energies $=-781.27737$ A.U.

$\begin{array}{lrrr}\text { C } & -1.83192700 & -0.57844200 & 1.34906600 \\ \mathrm{C} & -1.00354200 & -0.85784600 & 0.18552000 \\ \mathrm{C} & -1.56830100 & -0.22233400 & -1.15940600 \\ \mathrm{C} & -1.93080500 & 1.28313500 & -0.93344700 \\ \mathrm{C} & -2.97605100 & 1.43847200 & 0.18429500 \\ \mathrm{C} & -2.64272000 & 0.65473200 & 1.45694200 \\ \mathrm{H} & -3.10647900 & 2.49471900 & 0.43858500 \\ \mathrm{H} & -3.95091400 & 1.09786100 & -0.17705800 \\ \mathrm{H} & -2.04035800 & 1.26904700 & 2.15699200 \\ \mathrm{H} & -3.53815500 & 0.43063700 & 2.05345800 \\ \mathrm{C} & -1.82594500 & -1.43023100 & 2.56743800 \\ \mathrm{H} & -0.80984200 & -1.68941300 & 2.88381800 \\ \mathrm{H} & -2.36065100 & -0.96721700 & 3.39796400 \\ \mathrm{H} & -2.31353200 & -2.38816500 & 2.32937400 \\ \mathrm{C} & -0.73276000 & 2.28030100 & -0.79632400 \\ \mathrm{C} & -0.40414900 & -2.29715200 & 0.14718600 \\ \mathrm{C} & 0.25893300 & 2.02536800 & 0.31109700 \\ \mathrm{H} & -1.18485000 & 3.27220900 & -0.65337600 \\ \mathrm{H} & -0.21596900 & 2.33112100 & -1.75666500 \\ \mathrm{C} & 0.96814000 & -2.52578700 & -0.52258900 \\ \mathrm{C} & 2.11866600 & -1.59885400 & -0.16635300 \\ \mathrm{H} & 0.86313400 & -2.51438100 & -1.61050700 \\ \mathrm{H} & -2.43147200 & 1.59549600 & -1.85866500 \\ \mathrm{C} & -0.55074400 & -0.32316200 & -2.31187100 \\ \mathrm{H} & -0.46278900 & -1.34600200 & -2.68238800 \\ \mathrm{H} & -0.89884100 & 0.28755600 & -3.15155300 \\ \mathrm{H} & 0.44311700 & 0.02911600 & -2.02888800 \\ \mathrm{C} & -2.83477400 & -1.00973800 & -1.57644100 \\ \mathrm{H} & -3.27724600 & -0.56510100 & -2.47382400 \\ \mathrm{H} & -2.59441100 & -2.04881900 & -1.81859800 \\ \mathrm{H} & -3.60456600 & -1.02157900 & -0.79758900 \\ \mathrm{H} & -0.32053100 & -2.66387600 & 1.17055100 \\ \mathrm{H} & -1.13049800 & -2.95906300 & -0.33783200 \\ \mathrm{H} & 1.25838300 & -3.56024600 & -0.28758800 \\ \mathrm{C} & 2.15322100 & -0.87643600 & 0.97087500 \\ \mathrm{H} & 1.35439000 & -1.02932500 & 1.69871800 \\ \mathrm{C} & 3.12174600 & 0.20709900 & 1.35893700 \\ \mathrm{H} & 3.56832300 & -0.01038700 & 2.33730900 \\ \mathrm{H} & 3.94931800 & 0.27324700 & 0.64956600 \\ & & & \\ & & \end{array}$




$\begin{array}{lrrr}\mathrm{C} & 2.41403700 & 1.59759200 & 1.46741600 \\ \mathrm{H} & 1.75964200 & 1.59640800 & 2.34738100 \\ \mathrm{H} & 3.18677600 & 2.35552700 & 1.64905500 \\ \mathrm{C} & 1.60713700 & 1.95480900 & 0.23563700 \\ \mathrm{H} & -0.14316600 & -0.15277600 & 0.44768500 \\ \mathrm{H} & -0.16037100 & 1.95086600 & 1.31605000 \\ \mathrm{C} & 3.21124700 & -1.59704400 & -1.20975500 \\ \mathrm{H} & 3.54036700 & -2.62631900 & -1.40071200 \\ \mathrm{H} & 2.84726000 & -1.20794700 & -2.16915000 \\ \mathrm{H} & 4.09164400 & -1.02394900 & -0.91767900 \\ \mathrm{C} & 2.40822200 & 2.20202700 & -1.01637200 \\ \mathrm{H} & 3.07819700 & 3.05625400 & -0.85556500 \\ \mathrm{H} & 3.05002900 & 1.34867300 & -1.25920000 \\ \mathrm{H} & 1.79626300 & 2.42677900 & -1.89077800\end{array}$

Single point $E(m P W 1 P W 91)=-781.589378661$ A.U.

Structure 4:

No imaginary frequencies

$\mathrm{E}(\mathrm{b} 3$ lyp $)=-781.75798$ A.U.

Zero-point correction $=0.48420$ (Hartree/Particle)

Sum of electronic and zero-point Energies $=-781.27378$ A.U.

$\begin{array}{lrrr}\text { C } & 1.67300800 & 0.42220300 & 1.42425500 \\ \mathrm{C} & 1.28810000 & 0.93682000 & 0.22775600 \\ \mathrm{C} & 1.64422800 & 0.23039100 & -1.10525600 \\ \mathrm{C} & 1.89000200 & -1.30620000 & -0.90490800 \\ \mathrm{C} & 2.86535600 & -1.54718200 & 0.25927500 \\ \mathrm{C} & 2.41948300 & -0.88757500 & 1.56819700 \\ \mathrm{H} & 3.00515300 & -2.62295400 & 0.41323800 \\ \mathrm{H} & 3.84925300 & -1.15908500 & -0.02054700 \\ \mathrm{H} & 1.79430400 & -1.57363700 & 2.15902500 \\ \mathrm{H} & 3.29396100 & -0.70784000 & 2.20898800 \\ \mathrm{C} & 1.44752400 & 1.07880100 & 2.77263400 \\ \mathrm{H} & 0.60343900 & 1.77100700 & 2.81683000 \\ \mathrm{H} & 1.28237200 & 0.31422900 & 3.53971100 \\ \mathrm{H} & 2.33960200 & 1.63804800 & 3.08529200 \\ \mathrm{C} & 0.62943000 & -2.22905700 & -0.85129500 \\ \mathrm{C} & 0.74568100 & 2.36839800 & 0.17615200 \\ \mathrm{C} & -0.40872500 & -1.96027200 & 0.20839200 \\ \mathrm{H} & 1.01621900 & -3.24781500 & -0.69892900 \\ \mathrm{H} & 0.15971000 & -2.24595800 & -1.83867500 \\ \mathrm{C} & -0.67211600 & 2.71330300 & -0.37832200 \\ \mathrm{C} & -1.86065600 & 1.86055200 & -0.15560800\end{array}$




$\begin{array}{lrrr}\text { H } & -0.64649800 & 2.93506400 & -1.45248300 \\ \mathrm{H} & 2.40646200 & -1.63659600 & -1.81596300 \\ \mathrm{C} & 0.57766500 & 0.42559300 & -2.20885900 \\ \mathrm{H} & 0.52018100 & 1.46707800 & -2.53743200 \\ \mathrm{H} & 0.85463800 & -0.16076900 & -3.09111800 \\ \mathrm{H} & -0.42185100 & 0.09485700 & -1.90875700 \\ \mathrm{C} & 2.95122700 & 0.87376200 & -1.65399400 \\ \mathrm{H} & 3.25014000 & 0.39193400 & -2.59151400 \\ \mathrm{H} & 2.80953500 & 1.93782200 & -1.86901500 \\ \mathrm{H} & 3.77992500 & 0.79128200 & -0.94757700 \\ \mathrm{H} & 0.82858800 & 2.81187500 & 1.16734400 \\ \mathrm{H} & 1.42204300 & 2.96567800 & -0.44797900 \\ \mathrm{H} & -0.96886800 & 3.68825600 & 0.05749300 \\ \mathrm{C} & -1.85365900 & 0.80102800 & 0.81401200 \\ \mathrm{H} & -1.28841400 & 1.08151100 & 1.71131500 \\ \mathrm{C} & -3.06523200 & -0.09617400 & 1.08324900 \\ \mathrm{H} & -3.60729700 & 0.25066300 & 1.96934000 \\ \mathrm{H} & -3.76988100 & -0.05597400 & 0.24796200 \\ \mathrm{C} & -2.60552400 & -1.57129600 & 1.28314300 \\ \mathrm{H} & -2.03296500 & -1.64692600 & 2.21416600 \\ \mathrm{H} & -3.49516900 & -2.19913700 & 1.40152700 \\ \mathrm{C} & -1.75806000 & -2.02921600 & 0.10905600 \\ \mathrm{H} & -0.01974100 & -1.72649700 & 1.19567600 \\ \mathrm{C} & -3.01834400 & 2.07846300 & -1.05885200 \\ \mathrm{H} & -3.95524600 & 2.12679000 & -0.49362700 \\ \mathrm{H} & -2.90383800 & 2.96781700 & -1.68025800 \\ \mathrm{H} & -3.11579200 & 1.20729900 & -1.72513900 \\ \mathrm{C} & -2.51268100 & -2.46372600 & -1.12221700 \\ \mathrm{H} & -2.96417600 & -3.44868800 & -0.94493200 \\ \mathrm{H} & -3.34421900 & -1.78764200 & -1.35597500 \\ \mathrm{H} & -1.88216200 & -2.55079000 & -2.00879000 \\ \mathrm{H} & -1.05965500 & 0.13739700 & 0.31995000 \\ & & & \end{array}$

Single Point E(mPW1PW91)= -781.58591 A.U.

Structure 6:

No imaginary frequencies $\mathrm{E}(\mathrm{b} 3$ lyp $)=-781.76184$ A.U.

Zero-point correction $=0.48472($ Hartree/Particle $)$

Sum of electronic and zero-point Energies $=-781.27712$ A.U.
C
1.63390400
0.20080100
1.48066200
C $\quad 1.31690000$
0.86760600
0.33367700
C
1.76348100
0.30201800
$-1.03842400$ 


$\begin{array}{lrrr}\mathrm{C} & 1.83414800 & -1.26434200 & -1.01741300 \\ \mathrm{C} & 2.71229200 & -1.75159700 & 0.14721200 \\ \mathrm{C} & 2.30617800 & -1.15487200 & 1.50343300 \\ \mathrm{H} & 2.69433800 & -2.84649300 & 0.20112700 \\ \mathrm{H} & 3.75093400 & -1.48748500 & -0.06778700 \\ \mathrm{H} & 1.63669600 & -1.84443600 & 2.04486400 \\ \mathrm{H} & 3.18556000 & -1.08920800 & 2.15933500 \\ \mathrm{C} & 1.38728500 & 0.72037000 & 2.88370200 \\ \mathrm{H} & 0.53084300 & 1.39015900 & 2.97814300 \\ \mathrm{H} & 1.23416200 & -0.11282100 & 3.57825500 \\ \mathrm{H} & 2.26614800 & 1.26826600 & 3.24956800 \\ \mathrm{C} & 0.46312300 & -1.99777800 & -1.09453300 \\ \mathrm{C} & 0.77212600 & 2.29379100 & 0.41426200 \\ \mathrm{C} & -0.49092900 & -1.71513700 & 0.06891900 \\ \mathrm{H} & 0.66363300 & -3.07592400 & -1.13514500 \\ \mathrm{H} & -0.01931500 & -1.74424500 & -2.04284200 \\ \mathrm{C} & -0.59943600 & 2.69759600 & -0.21289100 \\ \mathrm{C} & -1.81492600 & 1.79433500 & -0.09627200 \\ \mathrm{H} & -0.46566500 & 2.91184100 & -1.27617700 \\ \mathrm{H} & 2.34406700 & -1.55345100 & -1.94564500 \\ \mathrm{C} & 0.86700900 & 0.74598000 & -2.21654400 \\ \mathrm{H} & 0.97301100 & 1.81387500 & -2.41936600 \\ \mathrm{H} & 1.17411100 & 0.21987700 & -3.12710700 \\ \mathrm{H} & -0.19445400 & 0.54081400 & -2.05397000 \\ \mathrm{C} & 3.18227800 & 0.86607000 & -1.33958300 \\ \mathrm{H} & 3.55706200 & 0.47627900 & -2.29317900 \\ \mathrm{H} & 3.15405500 & 1.95687800 & -1.42116800 \\ \mathrm{H} & 3.90349000 & 0.61682300 & -0.55863700 \\ \mathrm{H} & 0.76778000 & 2.60360600 & 1.45858500 \\ \mathrm{H} & 1.50738600 & 2.95514500 & -0.06426300 \\ \mathrm{H} & -0.86396600 & 3.66948400 & 0.22832500 \\ \mathrm{C} & -2.02190200 & 0.96048300 & 0.94724500 \\ \mathrm{H} & -1.29769700 & 0.96678400 & 1.75935400 \\ \mathrm{C} & -3.15604900 & -0.00265000 & 1.11275800 \\ \mathrm{H} & -3.66481400 & 0.14861900 & 2.07135100 \\ \mathrm{H} & -3.90648500 & 0.09562700 & 0.32775400 \\ \mathrm{C} & -2.70619000 & -1.54975700 & 1.18653000 \\ \mathrm{H} & -2.12413400 & -1.68480700 & 2.10157100 \\ \mathrm{H} & -3.62782400 & -2.13599700 & 1.24311600 \\ \mathrm{C} & -1.92160500 & -1.89162500 & -0.00934800 \\ \mathrm{H} & -0.45178700 & -0.59984500 & 0.30548800 \\ \mathrm{H} & -0.13017400 & -2.10825300 & 1.02815300 \\ \mathrm{C} & -2.78634100 & 1.97956500 & -1.23970200 \\ \mathrm{H} & -3.05878300 & 3.03955200 & -1.31627600 \\ \mathrm{H} & -2.32029300 & 1.71872000 & -2.19786500 \\ \mathrm{H} & -3.71394900 & 1.41433900 & -1.14185600 \\ & & & \end{array}$




$\begin{array}{llll}\mathrm{C} & -2.61244900 & -2.20298100 & -1.27841000 \\ \mathrm{H} & -2.30393900 & -3.20792000 & -1.60625500 \\ \mathrm{H} & -3.69946600 & -2.16993100 & -1.19907300 \\ \mathrm{H} & -2.27390000 & -1.53312300 & -2.08005200\end{array}$

Single point $E(m P W 1 P W 91)=-781.58890$ A.U.

Structure: TS3to4

1 imaginary frequency $=1259.639 \mathrm{~cm}-1$

$E($ b3lyp $)=-781.74062$ A.U.

Zero-point correction $=0.481214$ (Hartree/Particle)

Sum of electronic and zero-point Energies $=-781.25941 \mathrm{~A}$.U.

$\begin{array}{lrrr}\text { C } & 1.58967100 & 0.43256100 & 1.39977900 \\ \mathrm{C} & 1.02308500 & 0.89828900 & 0.21084600 \\ \mathrm{C} & 1.44588600 & 0.21838200 & -1.13772100 \\ \mathrm{C} & 1.84529700 & -1.29004300 & -0.95737300 \\ \mathrm{C} & 2.87520300 & -1.44457000 & 0.17395400 \\ \mathrm{C} & 2.43099100 & -0.80665100 & 1.49391200 \\ \mathrm{H} & 3.09701900 & -2.50395100 & 0.33803700 \\ \mathrm{H} & 3.81945000 & -0.98768400 & -0.13683800 \\ \mathrm{H} & 1.88908800 & -1.52254500 & 2.12973500 \\ \mathrm{H} & 3.30655900 & -0.54070900 & 2.10526700 \\ \mathrm{C} & 1.42751500 & 1.12187500 & 2.72805600 \\ \mathrm{H} & 0.46370100 & 1.61740100 & 2.86527100 \\ \mathrm{H} & 1.57161000 & 0.41622900 & 3.55031200 \\ \mathrm{H} & 2.19913300 & 1.89746100 & 2.83636200 \\ \mathrm{C} & 0.68681600 & -2.33275200 & -0.87208200 \\ \mathrm{C} & 0.65363000 & 2.40048200 & 0.17474500 \\ \mathrm{C} & -0.29929100 & -2.10917200 & 0.23735600 \\ \mathrm{H} & 1.17073900 & -3.31208700 & -0.74735700 \\ \mathrm{H} & 0.17531300 & -2.37939000 & -1.83681500 \\ \mathrm{C} & -0.74717100 & 2.79860100 & -0.38287900 \\ \mathrm{C} & -1.82756400 & 1.77969500 & -0.18724900 \\ \mathrm{H} & -0.69351000 & 3.06161100 & -1.44273500 \\ \mathrm{H} & 2.36789600 & -1.55290600 & -1.88572300 \\ \mathrm{C} & 0.35978700 & 0.31669000 & -2.23016100 \\ \mathrm{H} & 0.17179700 & 1.35246200 & -2.52375000 \\ \mathrm{H} & 0.70642900 & -0.20376800 & -3.12889300 \\ \mathrm{H} & -0.58329700 & -0.14313200 & -1.92952300 \\ \mathrm{C} & 2.69213700 & 0.98109700 & -1.67726400 \\ \mathrm{H} & 3.09769800 & 0.45859300 & -2.54946200 \\ \mathrm{H} & 2.43794100 & 1.99261600 & -2.00566700 \\ \mathrm{H} & 3.49001900 & 1.06434800 & -0.93413900 \\ & & & \\ & & & \end{array}$




$\begin{array}{llll}\mathrm{H} & 0.75079700 & 2.81406600 & 1.17699800 \\ \mathrm{H} & 1.40303000 & 2.92260500 & -0.42501800 \\ \mathrm{H} & -1.05276100 & 3.72148800 & 0.13045600 \\ \mathrm{C} & -1.77667700 & 0.94181500 & 0.91944700 \\ \mathrm{H} & -1.24882900 & 1.35545100 & 1.78330100 \\ \mathrm{C} & -2.84569800 & -0.08253500 & 1.28126700 \\ \mathrm{H} & -3.29334100 & 0.22914400 & 2.23279000 \\ \mathrm{H} & -3.65281800 & -0.05047300 & 0.54701800 \\ \mathrm{C} & -2.36994400 & -1.55399900 & 1.42648600 \\ \mathrm{H} & -1.71659800 & -1.64296100 & 2.30179000 \\ \mathrm{H} & -3.25795600 & -2.16428400 & 1.63146800 \\ \mathrm{C} & -1.64042800 & -2.03101100 & 0.18924100 \\ \mathrm{H} & -0.41426200 & 0.50083600 & 0.52514500 \\ \mathrm{H} & 0.13900000 & -1.96698400 & 1.22155100 \\ \mathrm{C} & -2.84290500 & 1.69984900 & -1.28143200 \\ \mathrm{H} & -3.28694600 & 2.69640100 & -1.40982000 \\ \mathrm{H} & -2.36327200 & 1.46165900 & -2.23789700 \\ \mathrm{H} & -3.64669300 & 0.98880300 & -1.10221800 \\ \mathrm{C} & -2.48891000 & -2.32676600 & -1.02216300 \\ \mathrm{H} & -3.14410600 & -3.18168300 & -0.81170900 \\ \mathrm{H} & -3.15055400 & -1.49153800 & -1.28182200 \\ \mathrm{H} & -1.90086700 & -2.57808800 & -1.90670700\end{array}$

Single point $E(m P W 1 P W 91)=-781.57059$ A.U.

Structure: TS3to6

1 imaginary frequency $=1120.45 \mathrm{~cm}-1$

$E($ b3lyp $)=-781.74991$ A.U.

Zero-point correction $=0.48180$ (Hartree/Particle)

Sum of electronic and zero-point Energies $=-781.26811$ A.U.

$\begin{array}{llll}\mathrm{C} & 1.63093600 & 0.34004300 & 1.47766900 \\ \mathrm{C} & 0.99779100 & 0.83603800 & 0.32915000 \\ \mathrm{C} & 1.66954900 & 0.52253500 & -1.05204800 \\ \mathrm{C} & 2.14799400 & -0.96299600 & -1.06023500 \\ \mathrm{C} & 3.15454500 & -1.22264800 & 0.07026500 \\ \mathrm{C} & 2.63866800 & -0.77096200 & 1.44281500 \\ \mathrm{H} & 3.41065800 & -2.28659000 & 0.10838400 \\ \mathrm{H} & 4.08883000 & -0.70096800 & -0.15379400 \\ \mathrm{H} & 2.18476000 & -1.61195300 & 1.99408100 \\ \mathrm{H} & 3.47044900 & -0.46848600 & 2.09536600 \\ \mathrm{C} & 1.30690600 & 0.80119100 & 2.87292200 \\ \mathrm{H} & 0.23314900 & 0.89563700 & 3.05660800 \\ \mathrm{H} & 1.72485400 & 0.12202200 & 3.61919000\end{array}$

S

8 


$\begin{array}{lrrr}\text { H } & 1.74267100 & 1.79431600 & 3.04792900 \\ \mathrm{C} & 0.97138900 & -1.97985000 & -1.06825900 \\ \mathrm{C} & 0.19847000 & 2.15110800 & 0.49329200 \\ \mathrm{C} & -0.06579100 & -1.79380100 & 0.03482900 \\ \mathrm{H} & 1.39486300 & -2.98806500 & -0.97464000 \\ \mathrm{H} & 0.48653000 & -1.94846500 & -2.04569500 \\ \mathrm{C} & -1.14100000 & 2.42428500 & -0.22574100 \\ \mathrm{C} & -2.28375100 & 1.44811700 & -0.01890900 \\ \mathrm{H} & -0.98523600 & 2.52761300 & -1.30258700 \\ \mathrm{H} & 2.67900800 & -1.11704000 & -2.00723200 \\ \mathrm{C} & 0.72443300 & 0.75514400 & -2.25125400 \\ \mathrm{H} & 0.58722100 & 1.81946100 & -2.45168300 \\ \mathrm{H} & 1.16925700 & 0.31815300 & -3.15154500 \\ \mathrm{H} & -0.26312800 & 0.31039200 & -2.11332900 \\ \mathrm{C} & 2.87952400 & 1.47592000 & -1.23713100 \\ \mathrm{H} & 3.41517900 & 1.22495200 & -2.15923100 \\ \mathrm{H} & 2.55009500 & 2.51474900 & -1.32808100 \\ \mathrm{H} & 3.59066600 & 1.42670300 & -0.40857900 \\ \mathrm{H} & 0.03625200 & 2.32910500 & 1.55611600 \\ \mathrm{H} & 0.88522600 & 2.94902000 & 0.18122300 \\ \mathrm{H} & -1.46345600 & 3.42431500 & 0.09946900 \\ \mathrm{C} & -2.29389300 & 0.57802700 & 1.00439600 \\ \mathrm{H} & -1.48127100 & 0.63649700 & 1.72667800 \\ \mathrm{C} & -3.18965000 & -0.61039600 & 1.20983600 \\ \mathrm{H} & -3.64619900 & -0.60988400 & 2.20651900 \\ \mathrm{H} & -4.00905800 & -0.63346300 & 0.48840100 \\ \mathrm{C} & -2.33531700 & -1.91830400 & 1.08890200 \\ \mathrm{H} & -1.73411600 & -2.04307500 & 1.99536200 \\ \mathrm{H} & -3.01318800 & -2.77992700 & 1.02559300 \\ \mathrm{C} & -1.43978900 & -1.89779700 & -0.12236600 \\ \mathrm{H} & 0.16506600 & -0.38394400 & 0.35911800 \\ \mathrm{H} & 0.27019700 & -2.07338300 & 1.03677800 \\ \mathrm{C} & -3.37475700 & 1.57009800 & -1.05613100 \\ \mathrm{H} & -3.73591900 & 2.60548400 & -1.09256400 \\ \mathrm{H} & -2.99990600 & 1.34105000 & -2.06186800 \\ \mathrm{H} & -4.23860600 & 0.93425100 & -0.85655300 \\ \mathrm{C} & -2.12384900 & -1.98068700 & -1.45030600 \\ \mathrm{H} & -2.33312700 & -3.04206000 & -1.65306800 \\ \mathrm{H} & -3.08949200 & -1.47012100 & -1.44451600 \\ \mathrm{H} & -1.52666900 & -1.60668600 & -2.28202700 \\ & & & \\ & & \end{array}$

Single point $E(m P W 1 P W 91)=-781.57059$ A.U. 
Structure: TS6to4

1 imaginary frequency $=993.276 \mathrm{~cm}-1$

$E($ b3lyp $)=-781.75047$ A.U.

Zero-point correction $=0.48140($ Hartree/Particle)

Sum of electronic and zero-point Energies $=-781.26907$ A.U.

$\begin{array}{lrrr}\mathrm{C} & 1.66631700 & 0.04362700 & 1.48713700 \\ \mathrm{C} & 1.45405600 & 0.76547200 & 0.35874700 \\ \mathrm{C} & 1.82949000 & 0.17942000 & -1.02376900 \\ \mathrm{C} & 1.63905100 & -1.37184100 & -1.04324600 \\ \mathrm{C} & 2.42622100 & -2.02651500 & 0.10532200 \\ \mathrm{C} & 2.15094200 & -1.39491600 & 1.48043200 \\ \mathrm{H} & 2.21480100 & -3.10155300 & 0.14043700 \\ \mathrm{H} & 3.49228700 & -1.94625500 & -0.12203400 \\ \mathrm{H} & 1.41410800 & -1.99558100 & 2.03715000 \\ \mathrm{H} & 3.05542100 & -1.45556800 & 2.10133500 \\ \mathrm{C} & 1.46501200 & 0.53671900 & 2.90658100 \\ \mathrm{H} & 0.91333800 & 1.47257400 & 3.00280200 \\ \mathrm{H} & 0.93806800 & -0.21979500 & 3.50227600 \\ \mathrm{H} & 2.43561300 & 0.68318600 & 3.39828100 \\ \mathrm{C} & 0.17300400 & -1.88094800 & -1.15465200 \\ \mathrm{C} & 1.06162400 & 2.23784600 & 0.44056000 \\ \mathrm{C} & -0.78810200 & -1.57635400 & -0.01909700 \\ \mathrm{H} & 0.23270900 & -2.97614900 & -1.23036600 \\ \mathrm{H} & -0.25531400 & -1.54969700 & -2.10535500 \\ \mathrm{C} & -0.28466000 & 2.73931700 & -0.17053000 \\ \mathrm{C} & -1.54201300 & 1.91546100 & -0.10628600 \\ \mathrm{H} & -0.14356600 & 3.03701800 & -1.21254200 \\ \mathrm{H} & 2.10122800 & -1.71803400 & -1.97741700 \\ \mathrm{C} & 1.04701900 & 0.79001500 & -2.20856100 \\ \mathrm{H} & 1.28936700 & 1.84618700 & -2.35238500 \\ \mathrm{H} & 1.33094600 & 0.27489300 & -3.13272600 \\ \mathrm{H} & -0.03828300 & 0.69825100 & -2.10963200 \\ \mathrm{C} & 3.32565600 & 0.51586100 & -1.28676000 \\ \mathrm{H} & 3.65428600 & 0.09770800 & -2.24540600 \\ \mathrm{H} & 3.47360400 & 1.59954900 & -1.33413800 \\ \mathrm{H} & 3.98073000 & 0.13383100 & -0.50158600 \\ \mathrm{H} & 1.10219500 & 2.56125800 & 1.47939200 \\ \mathrm{H} & 1.83498000 & 2.83167900 & -0.06363100 \\ \mathrm{H} & -0.53945800 & 3.68032900 & 0.34528600 \\ \mathrm{C} & -1.75139300 & 0.94072300 & 0.85772000 \\ \mathrm{H} & -1.03022000 & 0.92643400 & 1.67391100 \\ \mathrm{C} & -3.06985400 & 0.25190200 & 1.15589200 \\ \mathrm{H} & -3.43660700 & 0.57164400 & 2.13619100 \\ \mathrm{H} & -3.83903900 & 0.51554600 & 0.42776900 \\ \mathrm{C} & -2.91173400 & -1.31830900 & 1.18886300 \\ & & & \end{array}$




$\begin{array}{lrrr}\mathrm{H} & -2.36619000 & -1.60020000 & 2.09448000 \\ \mathrm{H} & -3.90655000 & -1.76837300 & 1.23576400 \\ \mathrm{C} & -2.16257100 & -1.73915400 & -0.03873100 \\ \mathrm{H} & -1.15193600 & -0.12498500 & 0.11074500 \\ \mathrm{H} & -0.34580400 & -1.56300500 & 0.97423900 \\ \mathrm{C} & -2.53034200 & 2.23273300 & -1.18805000 \\ \mathrm{H} & -2.74232500 & 3.30961400 & -1.17203400 \\ \mathrm{H} & -2.08762600 & 2.03257200 & -2.17165600 \\ \mathrm{H} & -3.47637500 & 1.69983700 & -1.11071400 \\ \mathrm{C} & -2.95651300 & -2.07227300 & -1.26199200 \\ \mathrm{H} & -3.33636400 & -3.09834500 & -1.15214700 \\ \mathrm{H} & -3.83987100 & -1.43129400 & -1.35941700 \\ \mathrm{H} & -2.37541700 & -2.03599300 & -2.18435700\end{array}$

Single point $E(m P W 1 P W 91)=-781.58066$ A.U. 
IRC 3 to 4

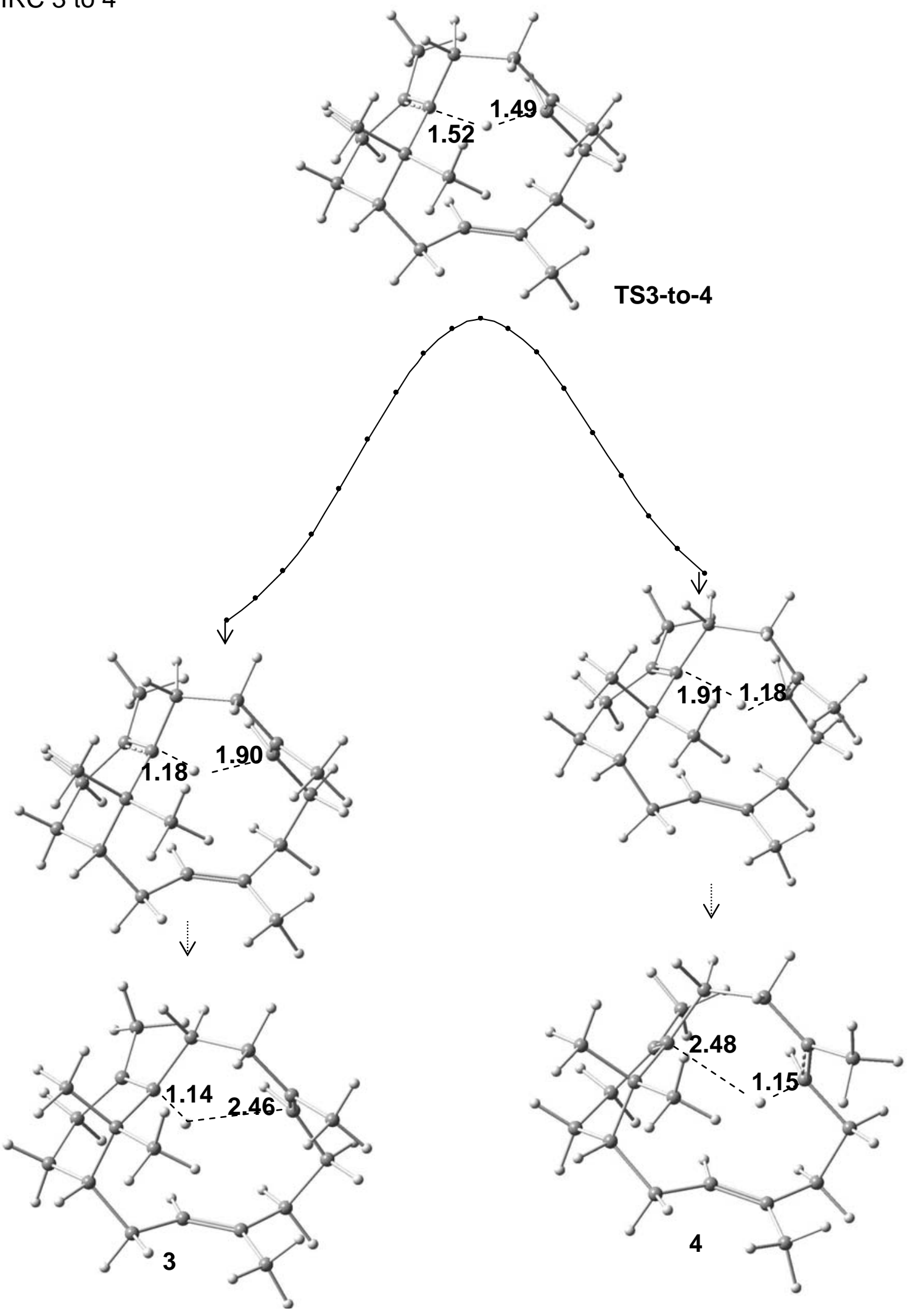




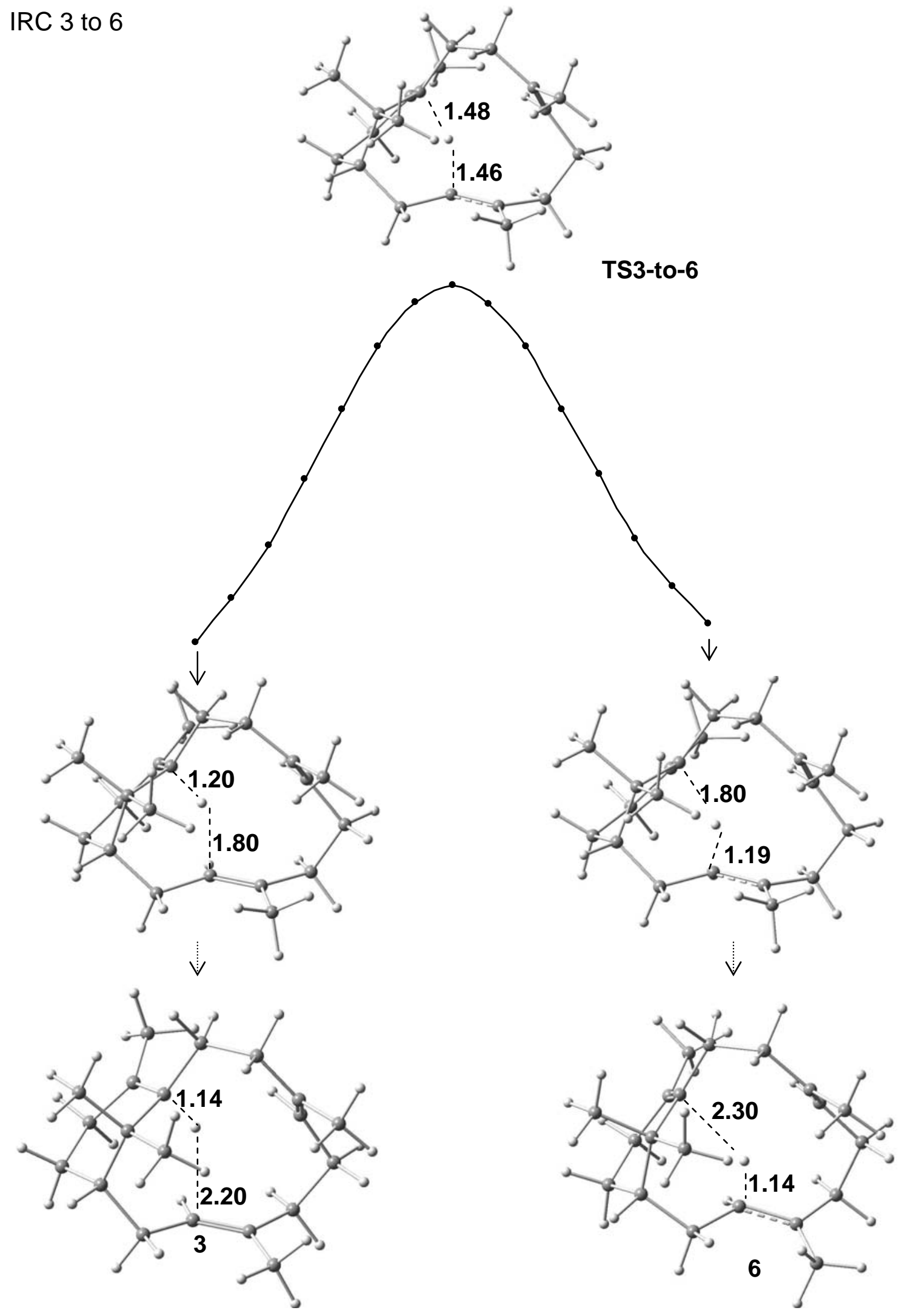




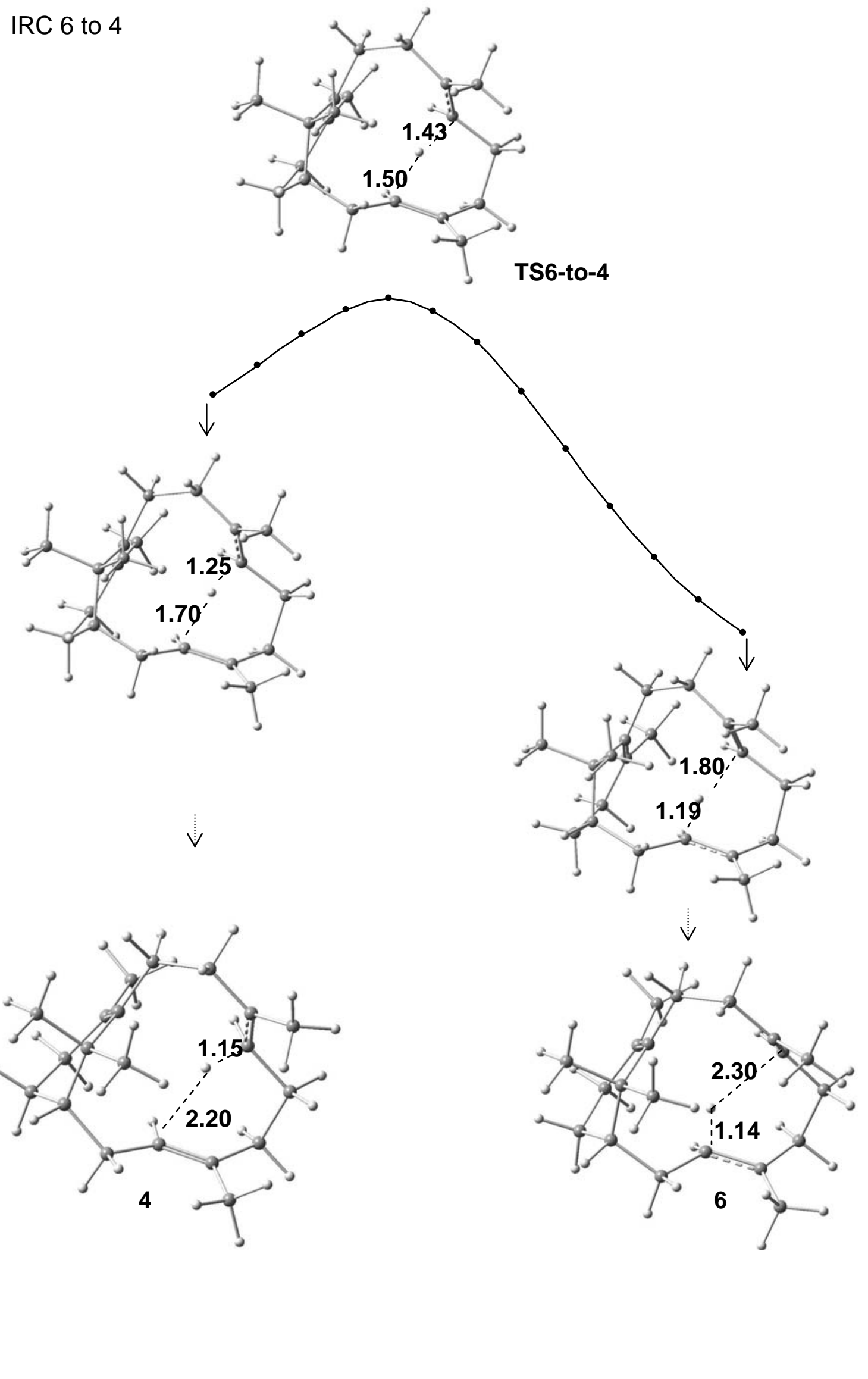


possible diversions from 6

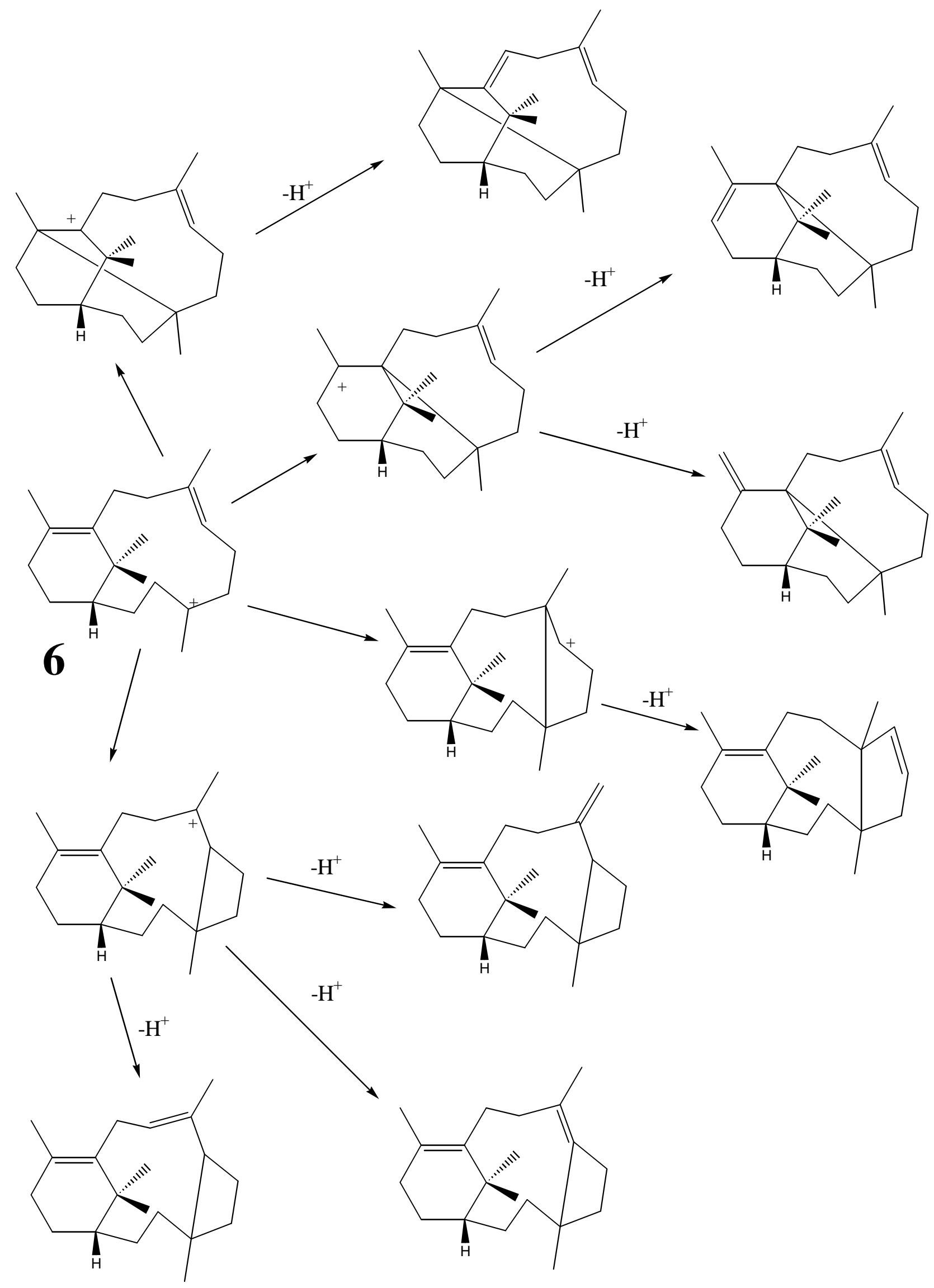


Note also that some natural products that could arise from cation 6 via somewhat circuitous routes have been described. See, for example:

- structure 74 in Hanson, J. R. Nat. Prod. Rep. 2002, 19, 125-132.

- structure 97 in Hanson, J. R. Nat. Prod. Rep. 2000, 17, 165-174.

- structure 118 in Hanson, J. R. Nat. Prod. Rep. 1996, 59-71.

- structure 166 in Hanson, J. R. Nat. Prod. Rep. 1994, 265-277.

- structure 126 in Hanson, J. R. Nat. Prod. Rep. 1998, 19, 93-106.

We will report on the various possible diversion pathways from 6 in a separate report. Note that most of the possible products from 6 suffer from considerable strain. Note also that proton transfers of the 4-to-6 variety, in which other (diastereotopic) protons are transferred, provide mechanisms for $E / Z$ isomerization. 
Conformers of 3 (energies compared to 3 ). Note that in all three, the lefthand $\mathrm{C}=\mathrm{C}$ double bond is closer to the migrating $\mathrm{H}$ than is the righthand one. Note also that the conformer discussed in the main text is the one that is "productive"-i.e. will lead directly to the experimentally observed stereoisomer of taxadiene.

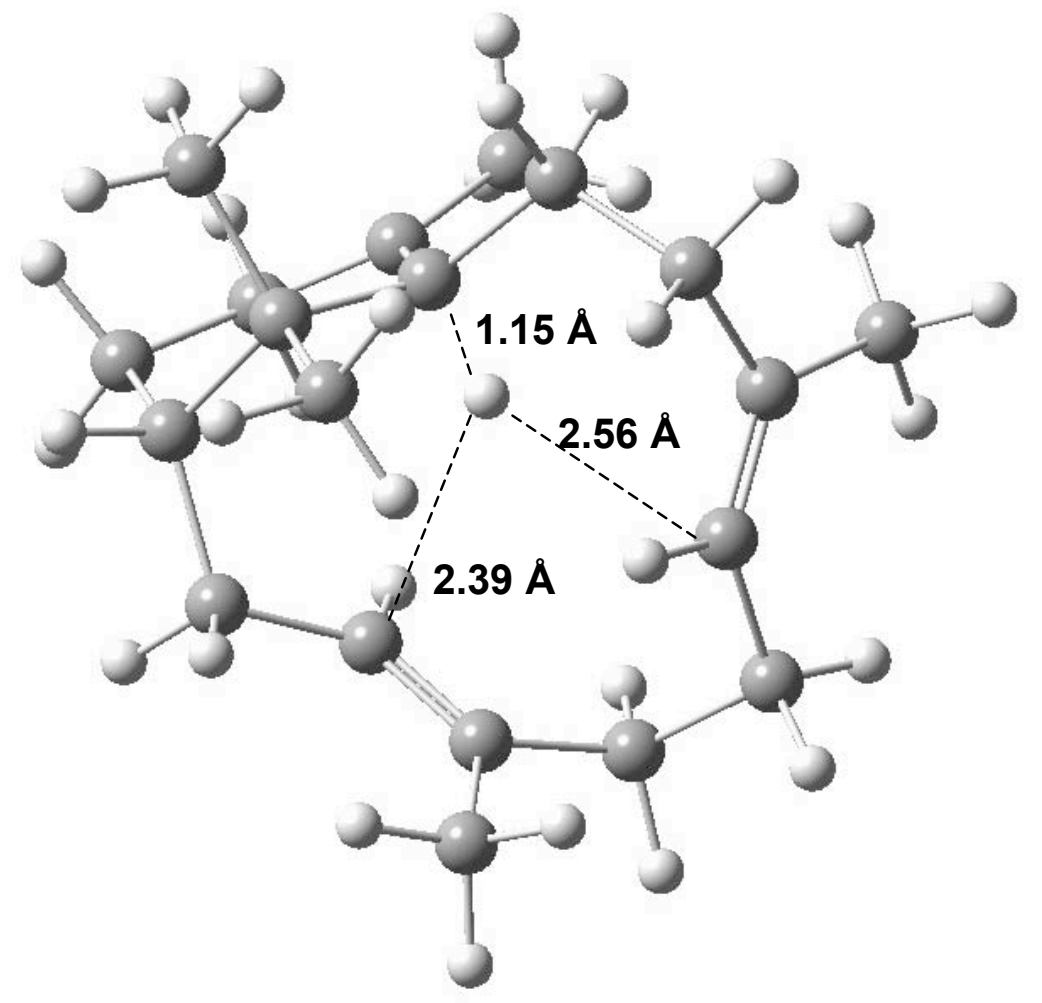

3-2

$\mathrm{S} 17$
$-1.56 \mathrm{kcal}$

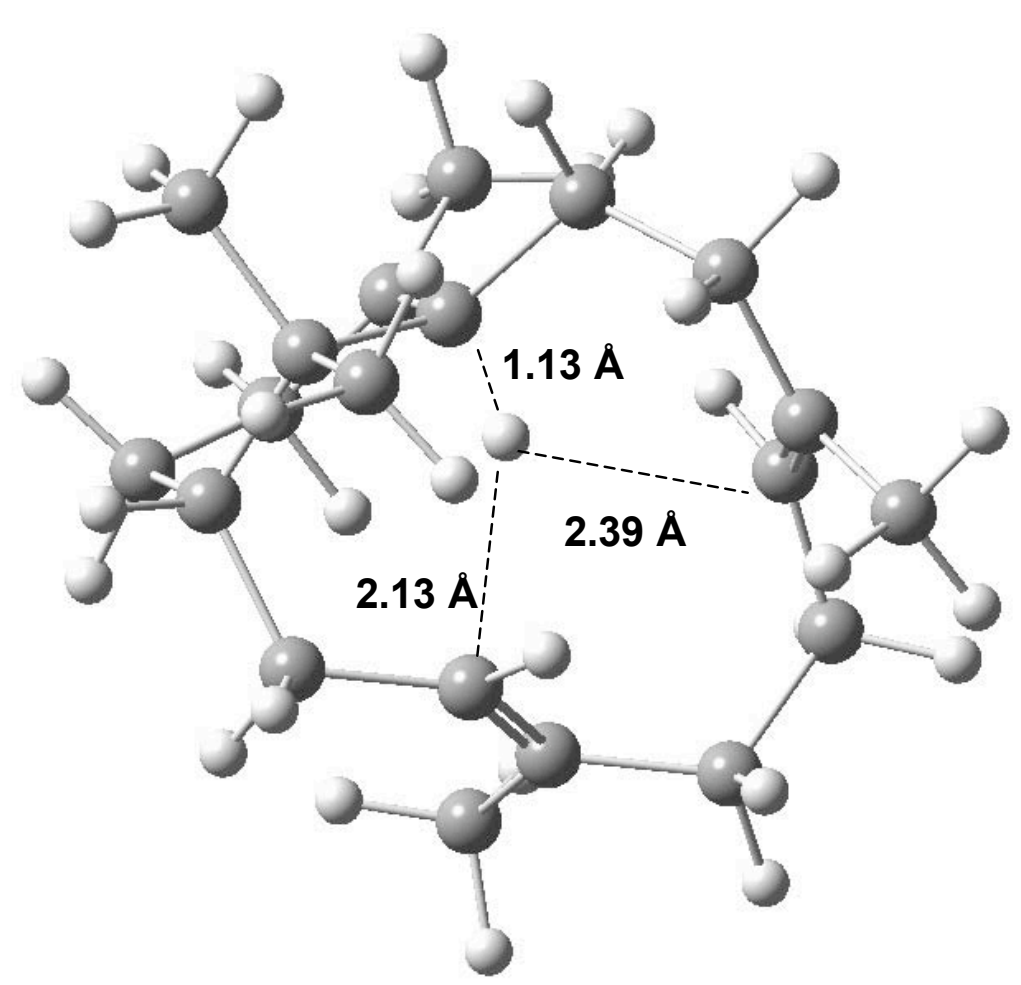

3-3

$3.44 \mathrm{kcal}$ 


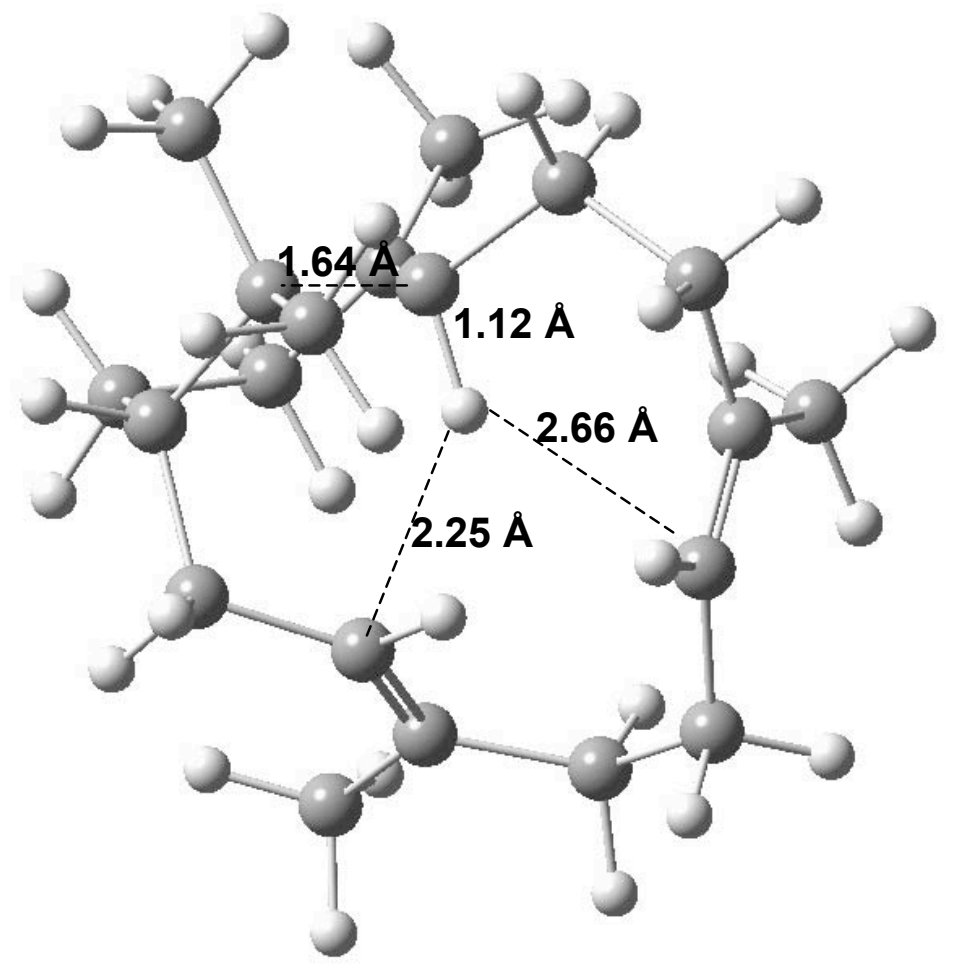

3-4

$2.88 \mathrm{kcal}$

S18 
Pathway from 3-2 to 4-2 directly and via 6-2 (energies in $\mathrm{kcal} / \mathrm{mol}$, relative to that of 3 from the main text). Note that again (although the differences are smaller, the 36-4 pathway has a lower overall barrier than does the direct 3-4 pathway.

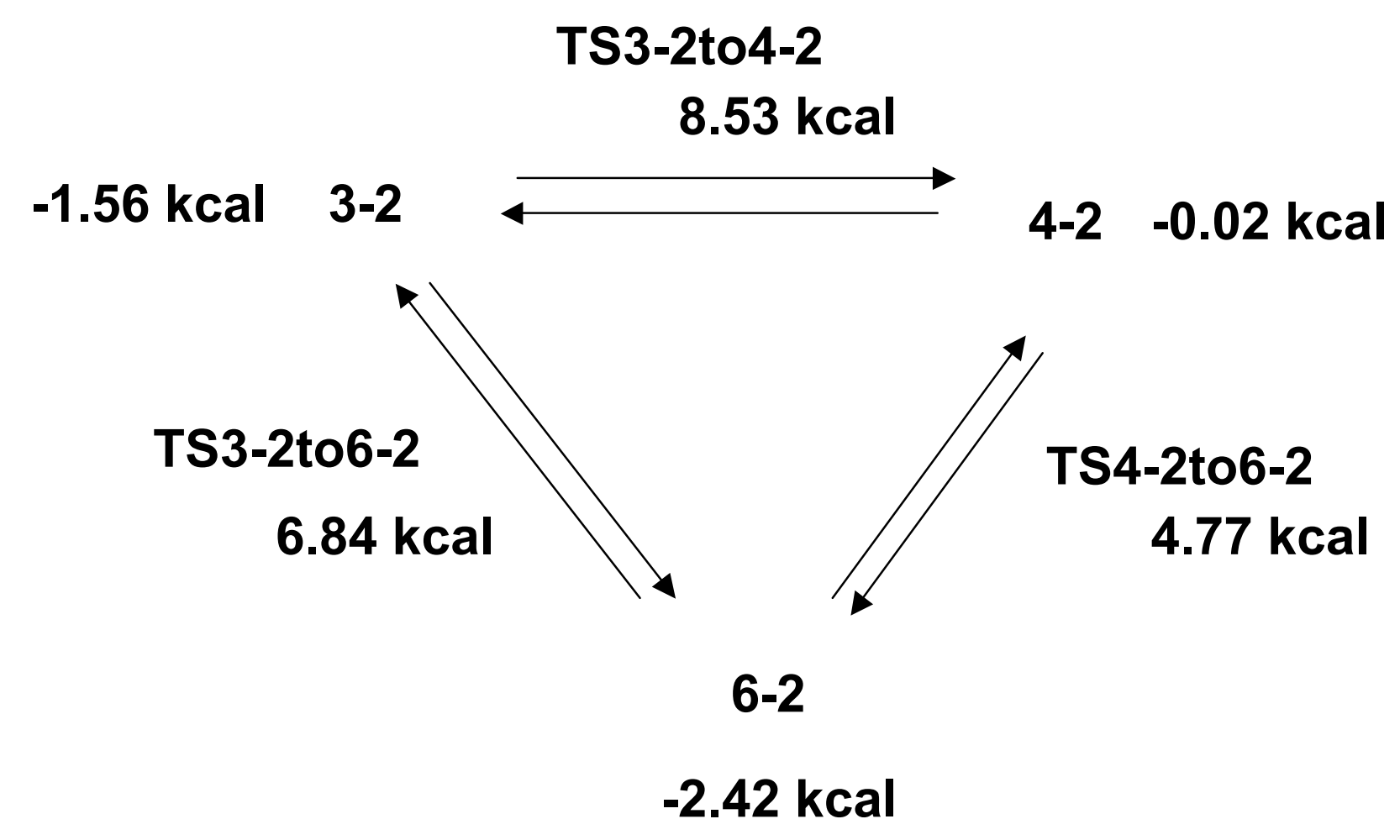

S19 


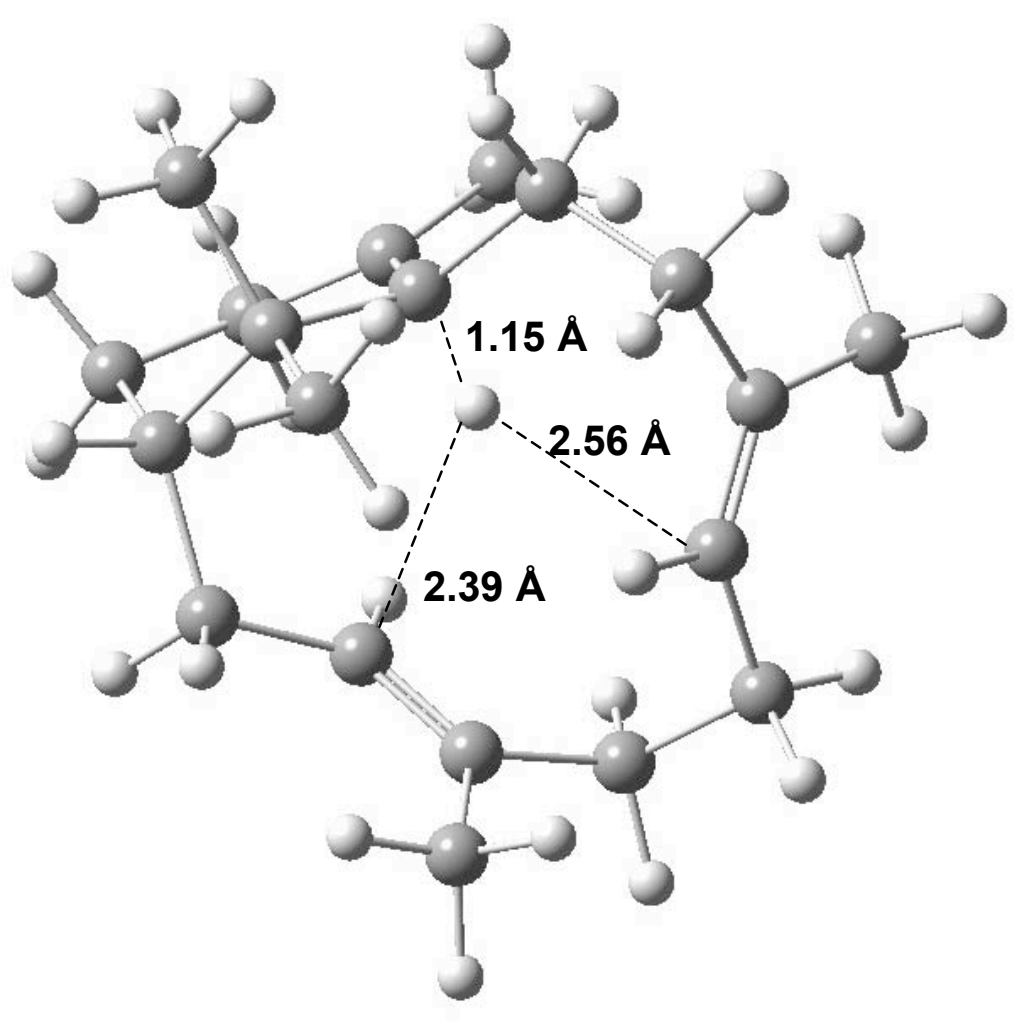

3-2

$-1.56 \mathrm{kcal}$

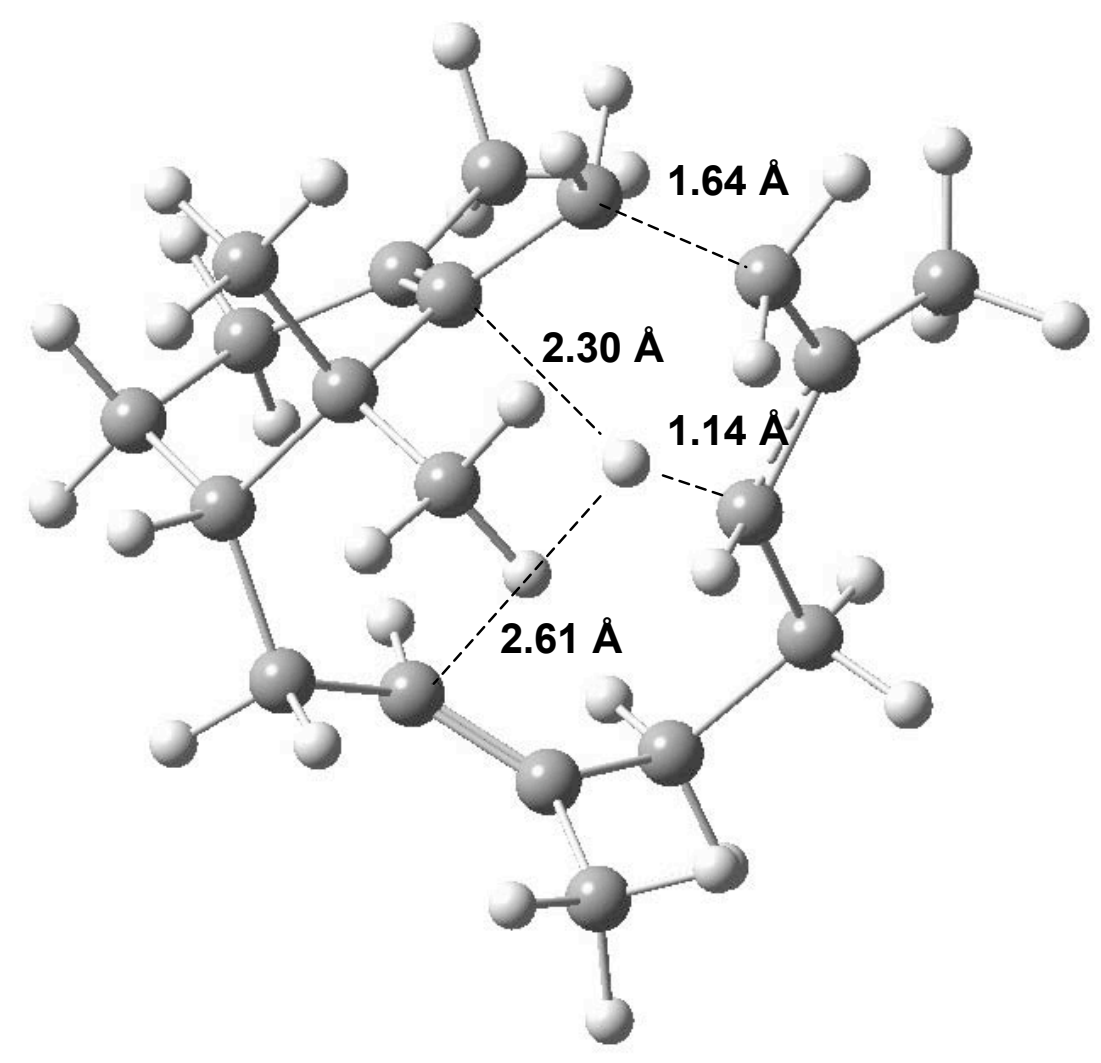

4-2

$-0.02 \mathrm{kcal}$

S20 


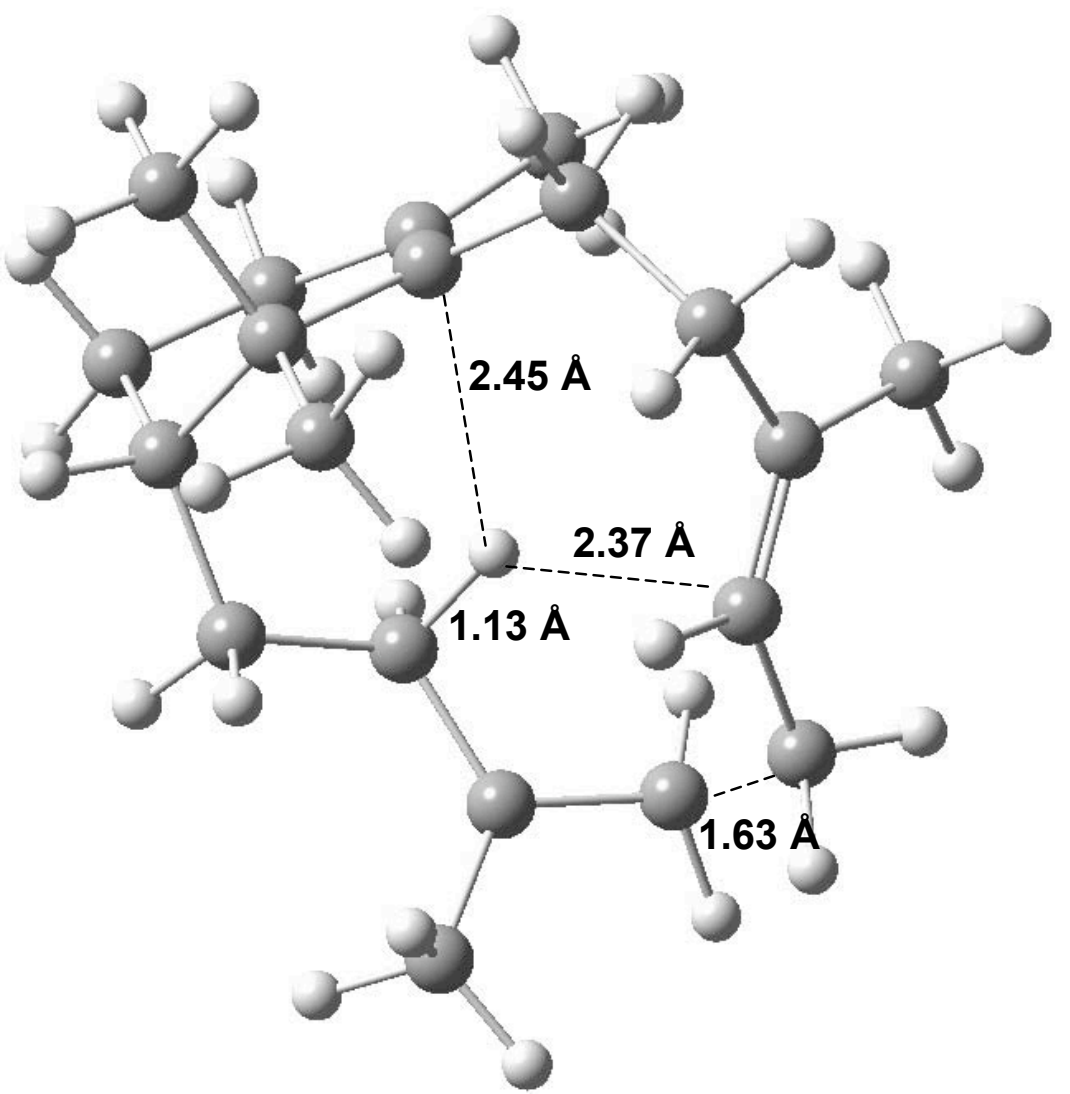

6-2

$-2.42 \mathrm{kcal}$

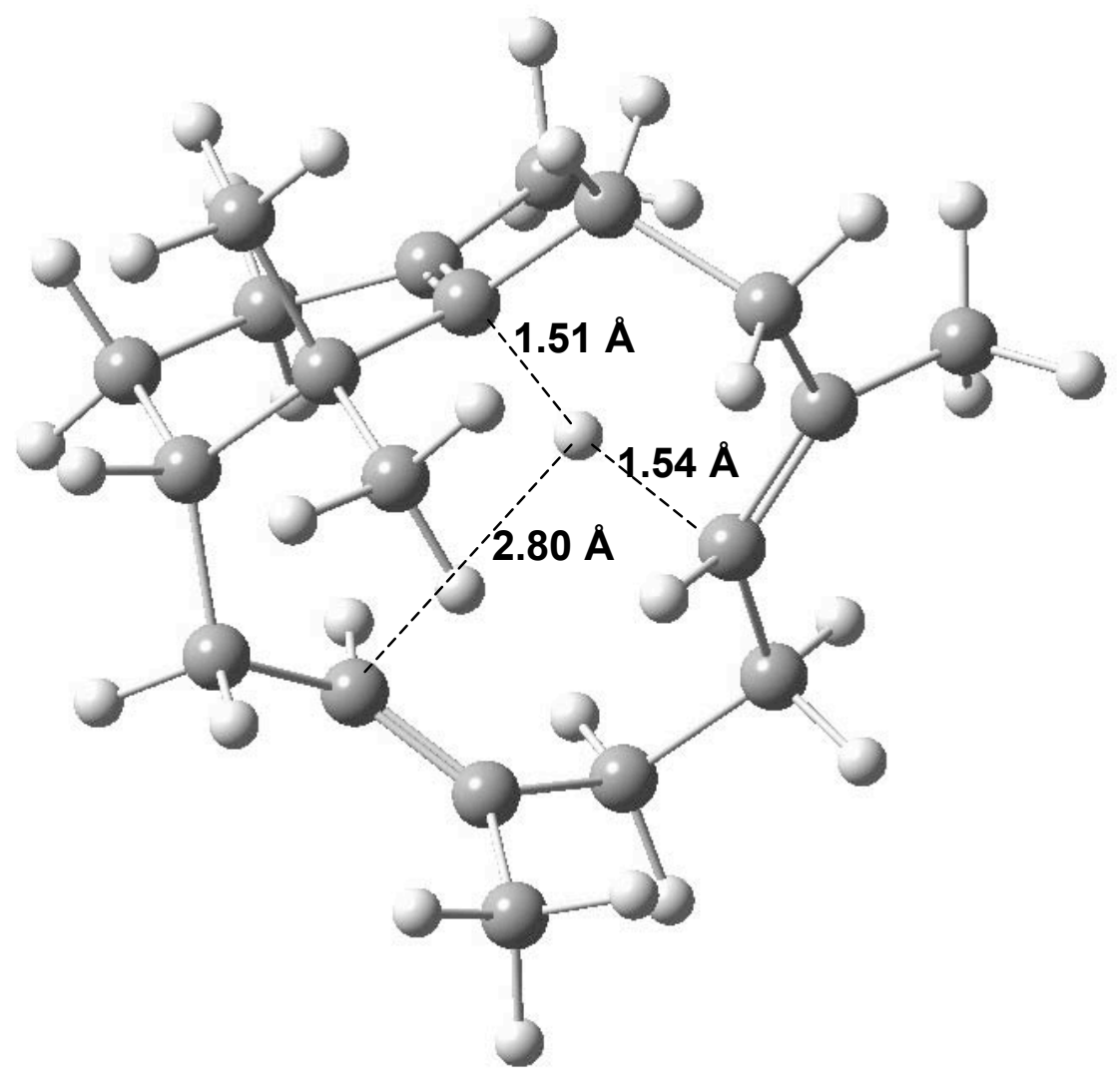

TS between 3-2 and 4-2

$8.53 \mathrm{kcal}$

S21 


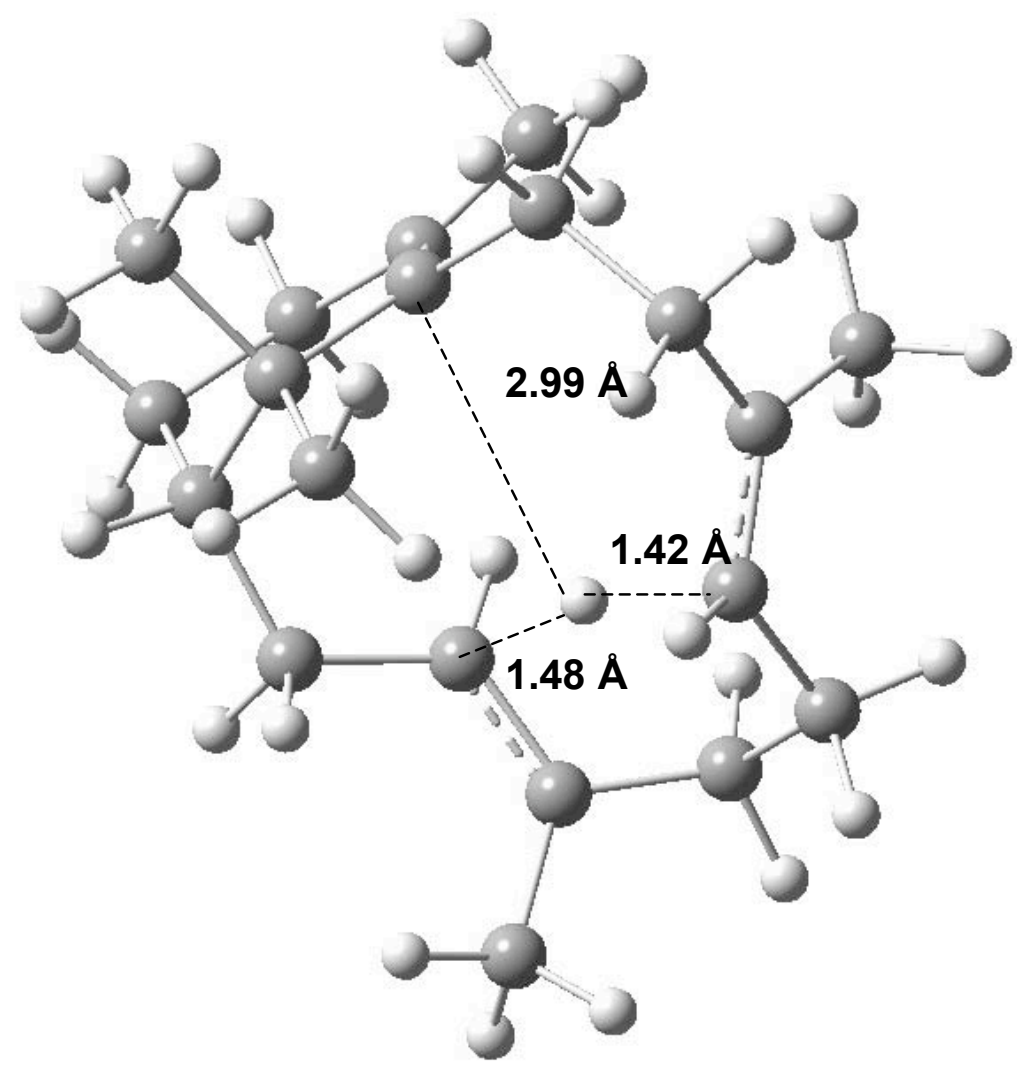

TS between 4-2 and 6-2

$4.77 \mathrm{kcal}$

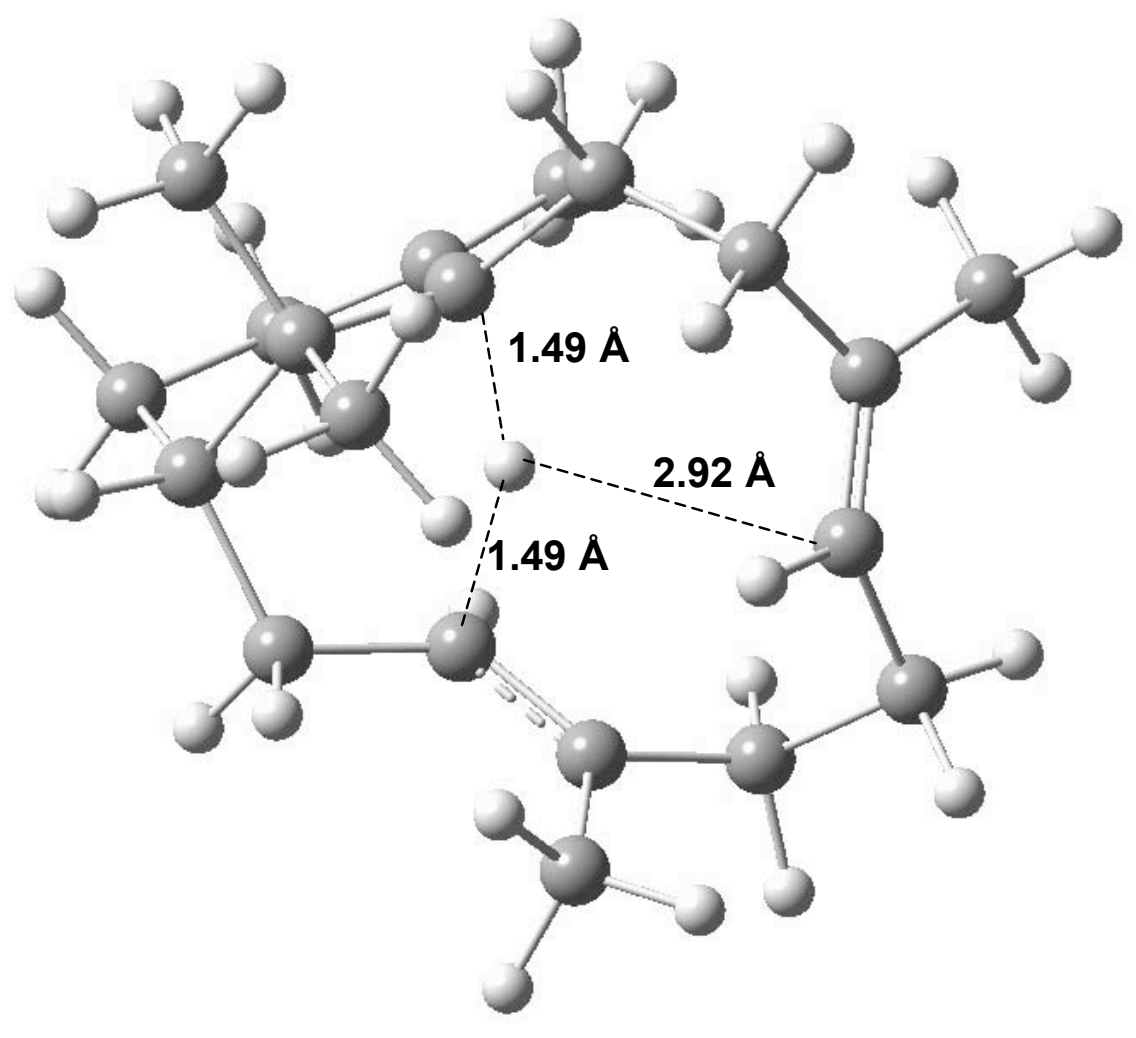

TS between 3-2 and 6-2 $6.84 \mathrm{kcal}$ 
Structure 3-2

No imaginary frequency

$E($ b3lyp $)=-781.76432$ A.U.

Zero-point correction $=0.484475$ (Hartree/Particle)

Sum of electronic and zero-point Energies= -781.27985 A.U.

$\begin{array}{lrrr}\mathrm{C} & -1.45847700 & 1.09296200 & -1.19024500 \\ \mathrm{C} & -0.96974300 & 0.89198100 & 0.16420000 \\ \mathrm{C} & -1.77625400 & -0.18068500 & 1.00606200 \\ \mathrm{C} & -2.07997800 & -1.46116900 & 0.15747500 \\ \mathrm{C} & -2.84579400 & -1.09390400 & -1.12663800 \\ \mathrm{C} & -2.20542600 & 0.04672200 & -1.92226900 \\ \mathrm{H} & -2.93957100 & -1.96985500 & -1.77577500 \\ \mathrm{H} & -3.86854100 & -0.80053100 & -0.87223100 \\ \mathrm{H} & -1.44427600 & -0.33841000 & -2.62962500 \\ \mathrm{H} & -2.91912800 & 0.54312500 & -2.59635300 \\ \mathrm{C} & -1.12369200 & 2.29897000 & -1.98817400 \\ \mathrm{H} & -0.11419000 & 2.66462100 & -1.78995500 \\ \mathrm{H} & -1.26669300 & 2.13646100 & -3.05819800 \\ \mathrm{H} & -1.80989100 & 3.10486700 & -1.68022600 \\ \mathrm{C} & -0.90100700 & -2.45802900 & -0.09027600 \\ \mathrm{C} & -0.50339700 & 2.17840300 & 0.92038300 \\ \mathrm{C} & 0.33615900 & -1.92416300 & -0.76620700 \\ \mathrm{H} & -1.32520100 & -3.27196600 & -0.69620600 \\ \mathrm{H} & -0.63564600 & -2.91649200 & 0.86492500 \\ \mathrm{C} & 0.93921100 & 2.15036500 & 1.50044100 \\ \mathrm{C} & 2.00847000 & 1.73411600 & 0.50747700 \\ \mathrm{H} & -1.96880000 & 1.47773400 & 2.36107100 \\ \mathrm{H} & -2.78429500 & -2.03999400 & 0.76805800 \\ \mathrm{C} & -1.00487700 & -0.57361300 & 2.27993500 \\ \mathrm{H} & -0.89465700 & 0.27277600 & 2.96270200 \\ \mathrm{H} & -1.56408900 & -1.34614400 & 2.81805400 \\ \mathrm{H} & -0.01122600 & -0.97084400 & 2.06320200 \\ \mathrm{C} & -3.11925200 & 0.45960900 & 1.44550500 \\ \mathrm{H} & -3.74125100 & -0.28579800 & 1.95098600 \\ \mathrm{H} & -2.96433300 & 1.27781800 & 2.15307000 \\ \mathrm{H} & -3.69676400 & 0.85887500 & 0.60481100 \\ \mathrm{H} & -0.59623800 & 3.04907900 & 0.26902200 \\ \mathrm{H} & -1.19970600 & 2.36204500 & 1.74294200 \\ \mathrm{H} & 1.14455600 & 3.15720200 & 1.88499500 \\ \mathrm{H} & 2.45332400 & 0.46237400 & 0.55050100 \\ \mathrm{H} & -1.04220100 & -0.15305000 & 1.34934500 \\ \mathrm{H} & -0.27117800 & -0.33432000 \\ \mathrm{H} & -14850000 & 0.39980700 & -1.00978000 \\ \mathrm{H} & -0.74261800 & 0.30129200 \\ \mathrm{H} & -1.37844200 & -1.18947800\end{array}$




$\begin{array}{lrrr}\mathrm{H} & 2.27715500 & -0.90659800 & -2.07653300 \\ \mathrm{H} & 3.48113800 & -2.07870900 & -1.54459600 \\ \mathrm{C} & 1.62954700 & -2.11447300 & -0.42948300 \\ \mathrm{H} & -0.00558000 & 0.36090000 & -0.15412500 \\ \mathrm{H} & 0.16955600 & -1.35160800 & -1.67937000 \\ \mathrm{C} & 2.51993500 & 2.79652600 & -0.43622600 \\ \mathrm{H} & 1.71203200 & 3.42236900 & -0.83358300 \\ \mathrm{H} & 3.18837100 & 3.48029500 & 0.10319800 \\ \mathrm{H} & 3.08051000 & 2.38814000 & -1.27985500 \\ \mathrm{C} & 2.10307400 & -2.99945100 & 0.69618400 \\ \mathrm{H} & 2.70346500 & -3.82145800 & 0.28609400 \\ \mathrm{H} & 2.75823500 & -2.45927500 & 1.38994200 \\ \mathrm{H} & 1.29115100 & -3.44429000 & 1.27362100\end{array}$

Structure 3-3

No imaginary frequency

$\mathrm{E}$ (b3lyp) $=-781.75700$ A.U.

Zero-point correction $=0.48510$ (Hartree/Particle)

Sum of electronic and zero-point Energies=-781.27190 A.U.

$\begin{array}{lrrr}\mathrm{C} & -1.39014600 & -0.11928800 & 1.57091100 \\ \mathrm{C} & -0.92234800 & -0.84167100 & 0.39846400 \\ \mathrm{C} & -1.86355700 & -0.57854500 & -0.87818400 \\ \mathrm{C} & -1.99094300 & 0.95881000 & -1.11423700 \\ \mathrm{C} & -2.56729500 & 1.65695200 & 0.12710900 \\ \mathrm{C} & -1.83945200 & 1.28177700 & 1.42714000 \\ \mathrm{H} & -2.53621800 & 2.74338700 & 0.00390800 \\ \mathrm{H} & -3.62392900 & 1.39989600 & 0.24859900 \\ \mathrm{H} & -0.86974600 & 1.82224500 & 1.46776800 \\ \mathrm{H} & -2.38485600 & 1.60995700 & 2.31685300 \\ \mathrm{C} & -1.32516300 & -0.65726100 & 2.95163600 \\ \mathrm{H} & -0.35953800 & -1.12025800 & 3.17800700 \\ \mathrm{H} & -1.56093000 & 0.09451200 & 3.70528600 \\ \mathrm{H} & -2.06121700 & -1.47449400 & 3.03041800 \\ \mathrm{C} & -0.72598000 & 1.69990200 & -1.68522700 \\ \mathrm{C} & -0.39713500 & -2.28386400 & 0.62766300 \\ \mathrm{C} & 0.66097300 & 1.33142200 & -1.20353500 \\ \mathrm{H} & -0.90276900 & 2.77243600 & -1.55448900 \\ \mathrm{H} & -0.72211800 & 1.53707000 & -2.76907400 \\ \mathrm{C} & 0.93055500 & -2.65895500 & -0.07472200 \\ \mathrm{C} & 2.10435300 & -1.69779600 & 0.03498900 \\ \mathrm{H} & 0.74774100 & -2.81576400 & -1.14390300 \\ \mathrm{H} & -2.74948400 & 1.06995300 & -1.89942800\end{array}$




$\begin{array}{lrrr}\mathrm{C} & -1.31457900 & -1.26815600 & -2.14023800 \\ \mathrm{H} & -1.34041800 & -2.35675700 & -2.04685900 \\ \mathrm{H} & -1.95245900 & -1.00622600 & -2.99107100 \\ \mathrm{H} & -0.29482900 & -0.97275000 & -2.38980100 \\ \mathrm{C} & -3.25277200 & -1.19724800 & -0.58663500 \\ \mathrm{H} & -3.93142400 & -1.01510100 & -1.42626500 \\ \mathrm{H} & -3.18291200 & -2.28156100 & -0.45925600 \\ \mathrm{H} & -3.72331800 & -0.79022500 & 0.31430300 \\ \mathrm{H} & -0.26764900 & -2.46308400 & 1.69598600 \\ \mathrm{H} & -1.16010600 & -3.00226300 & 0.31021200 \\ \mathrm{H} & 1.23018500 & -3.64708700 & 0.30155500 \\ \mathrm{C} & 2.16850000 & -0.70807600 & 0.94579600 \\ \mathrm{H} & 1.39075000 & -0.66624400 & 1.70404500 \\ \mathrm{C} & 3.13724900 & 0.45018100 & 0.97537500 \\ \mathrm{H} & 3.03761500 & 0.98065100 & 1.92848600 \\ \mathrm{H} & 4.17824500 & 0.11030500 & 0.92586100 \\ \mathrm{C} & 2.92707500 & 1.45486300 & -0.20786700 \\ \mathrm{H} & 3.61950500 & 2.29175300 & -0.05878000 \\ \mathrm{H} & 3.23314000 & 0.95446200 & -1.13094200 \\ \mathrm{C} & 1.51676300 & 1.99382400 & -0.38759100 \\ \mathrm{H} & -0.02627900 & -0.18969200 & 0.15658100 \\ \mathrm{H} & 1.08659800 & 0.46219400 & -1.70080300 \\ \mathrm{C} & 3.16497600 & -1.92174800 & -1.01625400 \\ \mathrm{H} & 3.43810500 & -2.98325300 & -1.05598000 \\ \mathrm{H} & 2.79938600 & -1.66043400 & -2.01871100 \\ \mathrm{H} & 4.07681100 & -1.35208300 & -0.82877000 \\ \mathrm{C} & 1.20620300 & 3.28098300 & 0.33542800 \\ \mathrm{H} & 1.42198800 & 3.19668900 & 1.40855700 \\ \mathrm{H} & 1.85491800 & 4.08300300 & -0.03839500 \\ \mathrm{H} & 0.17156300 & 3.61212800 & 0.21597600\end{array}$

Structure 3-4

No imaginary frequency

$\mathrm{E}($ b3lyp) $=-781.75802 \mathrm{~A} . \mathrm{U}$.

Zero-point correction $=0.48522$ (Hartree/Particle)

Sum of electronic and zero-point Energies= -781.27280 A.U.

$\begin{array}{lrrr}C & -1.29607200 & -0.39425200 & 1.49472300 \\ C & -0.97898500 & -0.84578400 & 0.14411400 \\ C & -1.90276400 & -0.04400000 & -0.94379500 \\ C & -1.76377500 & 1.49951500 & -0.73506700\end{array}$




\begin{tabular}{|c|c|c|c|}
\hline$C$ & -2.08402000 & 1.89532300 & 0.71346200 \\
\hline C & -1.29581100 & 1.05922100 & 1.75375900 \\
\hline $\mathrm{H}$ & -1.85781100 & 2.95185000 & 0.88179900 \\
\hline $\mathrm{H}$ & -3.15096800 & 1.77614700 & 0.92400200 \\
\hline $\mathrm{H}$ & -0.23045700 & 1.34387900 & 1.66266200 \\
\hline $\mathrm{H}$ & -1.61116100 & 1.28621500 & 2.77483600 \\
\hline C & -1.61964700 & -1.27456300 & 2.63796000 \\
\hline $\mathrm{H}$ & -1.20971600 & -2.28128400 & 2.58142300 \\
\hline $\mathrm{H}$ & -1.37841400 & -0.80771200 & 3.59582200 \\
\hline $\mathrm{H}$ & -2.72105400 & -1.38310000 & 2.61817600 \\
\hline C & -0.45178800 & 2.18811900 & -1.25746500 \\
\hline C & -0.80516000 & -2.36844800 & -0.08877800 \\
\hline C & 0.86505300 & 1.54654800 & -0.89366900 \\
\hline $\mathrm{H}$ & -0.49514700 & 3.23208900 & -0.93108400 \\
\hline $\mathrm{H}$ & -0.50878400 & 2.21843200 & -2.35129900 \\
\hline C & 0.54563700 & -2.77085000 & -0.75858700 \\
\hline C & 1.73950200 & -2.01023400 & -0.21279100 \\
\hline $\mathrm{H}$ & 0.48330600 & -2.60761700 & -1.83831300 \\
\hline $\mathrm{H}$ & -2.56185500 & 1.93678800 & -1.34997300 \\
\hline C & -1.49521500 & -0.44419800 & -2.37176600 \\
\hline $\mathrm{H}$ & -1.71893700 & -1.49428100 & -2.57407900 \\
\hline $\mathrm{H}$ & -2.07587900 & 0.14948200 & -3.08583000 \\
\hline $\mathrm{H}$ & -0.43841500 & -0.27701400 & -2.58389300 \\
\hline C & -3.37157700 & -0.47882000 & -0.73323600 \\
\hline $\mathrm{H}$ & -4.01265400 & -0.00484800 & -1.48349100 \\
\hline $\mathrm{H}$ & -3.48877800 & -1.56013500 & -0.84758300 \\
\hline $\mathrm{H}$ & -3.76251900 & -0.20668100 & 0.25212900 \\
\hline $\mathrm{H}$ & -0.87814100 & -2.90355200 & 0.85871200 \\
\hline $\mathrm{H}$ & -1.62540100 & -2.76004700 & -0.69669800 \\
\hline $\mathrm{H}$ & 0.66843400 & -3.85148800 & -0.61173300 \\
\hline C & 2.36031200 & -1.11770300 & -1.00673900 \\
\hline $\mathrm{H}$ & 2.02943700 & -1.09289300 & -2.04479000 \\
\hline C & 3.42821500 & -0.10075000 & -0.65972800 \\
\hline $\mathrm{H}$ & 4.29726700 & -0.59786500 & -0.21011400 \\
\hline $\mathrm{H}$ & 3.79028900 & 0.34334700 & -1.59268900 \\
\hline C & 3.02567200 & 1.04487000 & 0.30753300 \\
\hline $\mathrm{H}$ & 2.91974900 & 0.63751000 & 1.32527100 \\
\hline $\mathrm{H}$ & 3.88410400 & 1.72691900 & 0.37697700 \\
\hline C & 1.77998900 & 1.88000300 & 0.04370200 \\
\hline $\mathrm{H}$ & 0.01096300 & -0.34981300 & -0.02372800 \\
\hline $\mathrm{H}$ & 1.11757900 & 0.70295100 & -1.52217700 \\
\hline C & 2.11581300 & -2.26224000 & 1.22638700 \\
\hline $\mathrm{H}$ & 1.36541400 & -1.87302000 & 1.93062500 \\
\hline $\mathrm{H}$ & 2.18993700 & -3.33933600 & 1.41939600 \\
\hline $\mathrm{H}$ & 3.07373800 & -1.80904400 & 1.49059000 \\
\hline C & 1.68059100 & 3.10024900 & 0.93192000 \\
\hline
\end{tabular}




$\begin{array}{llll}\mathrm{H} & 1.81818100 & 2.84204700 & 1.98965800 \\ \mathrm{H} & 2.48642500 & 3.80285600 & 0.68563500 \\ \mathrm{H} & 0.73545700 & 3.63718100 & 0.82813000\end{array}$

Structure 4-2

No imaginary freqency

$E($ b3lyp $)=-781.76263$ A.U.

Zero-point correction $=0.48523$ (Hartree/Particle)

Sum of electronic and zero-point Energies $=-781.27740$ A.U.

$\begin{array}{lrrr}\mathrm{C} & -0.99865300 & -1.12969400 & 1.29790600 \\ \mathrm{C} & -0.99274500 & -1.16007700 & -0.06955900 \\ \mathrm{C} & -1.86787700 & -0.19549100 & -0.90836200 \\ \mathrm{C} & -2.29867000 & 1.08537900 & -0.10412100 \\ \mathrm{C} & -2.81848400 & 0.69298800 & 1.29230600 \\ \mathrm{C} & -1.84354600 & -0.17265800 & 2.10083600 \\ \mathrm{H} & -3.07065300 & 1.59369100 & 1.86218700 \\ \mathrm{H} & -3.75791800 & 0.14449200 & 1.17461100 \\ \mathrm{H} & -1.17151600 & 0.44437500 & 2.71339600 \\ \mathrm{H} & -2.40100700 & -0.76808100 & 2.83836400 \\ \mathrm{C} & -0.22932600 & -2.10514700 & 2.16179000 \\ \mathrm{H} & 0.71670900 & -2.43964000 & 1.72743800 \\ \mathrm{H} & -0.00803100 & -1.65995200 & 3.13636800 \\ \mathrm{H} & -0.82851200 & -3.00487100 & 2.35707500 \\ \mathrm{C} & -1.32412100 & 2.30946900 & -0.07691600 \\ \mathrm{C} & -0.32694400 & -2.32265200 & -0.76297900 \\ \mathrm{C} & 0.00511600 & 2.13514800 & 0.60860700 \\ \mathrm{H} & -1.88187000 & 3.10482400 & 0.43900900 \\ \mathrm{H} & -1.18300800 & 2.67283600 & -1.09832400 \\ \mathrm{C} & 1.14765700 & -2.16398400 & -1.44955900 \\ \mathrm{C} & 2.15021900 & -1.60020200 & -0.54229400 \\ \mathrm{H} & 1.00835600 & -1.50869700 & -2.31105800 \\ \mathrm{H} & -3.16632000 & 1.47575100 & -0.65154300 \\ \mathrm{C} & -1.18768000 & 0.22092400 & -2.23221000 \\ \mathrm{H} & -1.01526900 & -0.63820200 & -2.88880100 \\ \mathrm{H} & -1.84495600 & 0.90231200 & -2.78206300 \\ \mathrm{H} & -0.23910900 & 0.74170900 & -2.08185900 \\ \mathrm{C} & -3.16032700 & -0.97201400 & -1.30325900 \\ \mathrm{H} & -3.81662300 & -0.33121700 & -1.90150700 \\ \mathrm{H} & -2.93074200 & -1.85348700 & -1.91054400 \\ \mathrm{H} & -3.72139200 & -1.31414700 & -0.43031400 \\ \mathrm{H} & -0.24849900 & -3.18058100 & -0.09565700 \\ \mathrm{H} & -0.92835400 & -2.64086500 & -1.61755400\end{array}$




$\begin{array}{lrrr}\text { H } & 1.41832400 & -3.16758800 & -1.78492100 \\ \mathrm{C} & 2.02321300 & -0.18662600 & -0.26857000 \\ \mathrm{H} & 1.61609200 & 0.31605900 & -1.14828500 \\ \mathrm{C} & 3.07938700 & 0.69138900 & 0.40691300 \\ \mathrm{H} & 3.60369100 & 0.16411700 & 1.21015000 \\ \mathrm{H} & 3.83583600 & 0.94468100 & -0.34461600 \\ \mathrm{C} & 2.43386400 & 1.99134700 & 0.99224800 \\ \mathrm{H} & 2.11023300 & 1.78203100 & 2.01742200 \\ \mathrm{H} & 3.21002700 & 2.76220400 & 1.05594700 \\ \mathrm{C} & 1.23881800 & 2.46946000 & 0.18363400 \\ \mathrm{H} & 1.07166100 & -0.23447200 & 0.36041800 \\ \mathrm{H} & -0.04334900 & 1.67815100 & 1.59449200 \\ \mathrm{C} & 3.14249400 & -2.47234900 & 0.12177700 \\ \mathrm{H} & 2.81646200 & -3.51354000 & 0.18544200 \\ \mathrm{H} & 4.04200700 & -2.46242200 & -0.51995400 \\ \mathrm{H} & 3.46309000 & -2.09721700 & 1.09639400 \\ \mathrm{C} & 1.55531000 & 3.19812500 & -1.09862200 \\ \mathrm{H} & 2.05598100 & 4.14839500 & -0.87376300 \\ \mathrm{H} & 2.25305200 & 2.63194200 & -1.73178500 \\ \mathrm{H} & 0.66939000 & 3.42738700 & -1.69356200\end{array}$

Structure 6-2

No imaginary freqency

$E($ b3lyp $)=-781.76657$ A.U.

Zero-point correction $=0.48534$ (Hartree/Particle)

Sum of electronic and zero-point Energies= -781.28123 A.U.

$\begin{array}{lrrr}\mathrm{C} & 1.51024500 & 0.78573200 & 1.29151700 \\ \mathrm{C} & 1.39775200 & 0.86776000 & -0.06315400 \\ \mathrm{C} & 1.89561800 & -0.29788600 & -0.95877300 \\ \mathrm{C} & 1.79369700 & -1.68350000 & -0.23026500 \\ \mathrm{C} & 2.47026200 & -1.62110900 & 1.14772600 \\ \mathrm{C} & 1.96262600 & -0.46462200 & 2.01853100 \\ \mathrm{H} & 2.34301600 & -2.57345100 & 1.67636600 \\ \mathrm{H} & 3.54768700 & -1.50966200 & 0.99941200 \\ \mathrm{H} & 1.12735100 & -0.79624100 & 2.66029600 \\ \mathrm{H} & 2.74044300 & -0.18320600 & 2.74054200 \\ \mathrm{C} & 1.20879800 & 1.89686600 & 2.27304200 \\ \mathrm{H} & 0.92454100 & 2.84492600 & 1.82097200 \\ \mathrm{H} & 0.40546100 & 1.59915400 & 2.96116300 \\ \mathrm{H} & 2.08814500 & 2.08566400 & 2.90101100 \\ \mathrm{C} & 0.37706900 & -2.32673000 & -0.18254500\end{array}$




$\begin{array}{lrrr}\mathrm{C} & 1.01212600 & 2.17712000 & -0.74574000 \\ \mathrm{C} & -0.70069400 & -1.52972600 & 0.55855400 \\ \mathrm{H} & 0.48122500 & -3.31472800 & 0.28439200 \\ \mathrm{H} & 0.04626900 & -2.51225600 & -1.20924300 \\ \mathrm{C} & -0.41086100 & 2.33225500 & -1.39491100 \\ \mathrm{C} & -1.60291200 & 1.96286800 & -0.53930000 \\ \mathrm{H} & -0.45194400 & 1.75575800 & -2.32062200 \\ \mathrm{H} & 2.37726900 & -2.38443600 & -0.84164800 \\ \mathrm{C} & 1.17215600 & -0.39001300 & -2.32185200 \\ \mathrm{H} & 1.35333900 & 0.49606600 & -2.93524600 \\ \mathrm{H} & 1.55987600 & -1.24797300 & -2.88218000 \\ \mathrm{H} & 0.09061500 & -0.51633500 & -2.23196500 \\ \mathrm{C} & 3.39074100 & -0.01577900 & -1.28938600 \\ \mathrm{H} & 3.80425700 & -0.81464300 & -1.91563000 \\ \mathrm{H} & 3.49166300 & 0.92116400 & -1.84546100 \\ \mathrm{H} & 4.00583500 & 0.07245700 & -0.39158100 \\ \mathrm{H} & 1.15300400 & 3.00336600 & -0.04843000 \\ \mathrm{H} & 1.72028300 & 2.37119100 & -1.56015800 \\ \mathrm{H} & -0.49453600 & 3.38810900 & -1.68308400 \\ \mathrm{C} & -2.28873500 & 0.83525000 & -0.83638900 \\ \mathrm{H} & -1.93825600 & 0.26846800 & -1.69876900 \\ \mathrm{C} & -3.45607800 & 0.23217300 & -0.13258000 \\ \mathrm{H} & -4.04092000 & 0.95919100 & 0.43506500 \\ \mathrm{H} & -4.12642700 & -0.25308900 & -0.84679800 \\ \mathrm{C} & -3.06762300 & -0.89279000 & 0.98515400 \\ \mathrm{H} & -2.63798600 & -0.35677500 & 1.83421500 \\ \mathrm{H} & -2.00745700 & -1.37317600 & 1.26876000 \\ \mathrm{C} & -2.11536600 & -1.83396100 & 0.39966300 \\ \mathrm{H} & -0.63894600 & -0.43760900 & 0.29129200 \\ \mathrm{H} & -0.50549200 & -1.45267700 & 1.64085500 \\ \mathrm{C} & -1.98228100 & 2.91635000 & 0.56184700 \\ \mathrm{H} & -1.12710800 & 3.15376000 & 1.20039200 \\ \mathrm{H} & -2.31254600 & 3.86610600 & 0.12184800 \\ \mathrm{H} & -2.78831400 & 2.54923500 & 1.20056700 \\ \mathrm{C} & -2.59262300 & -2.93407000 & -0.46192100 \\ \mathrm{H} & -2.14172000 & -3.88104200 & -0.13122700 \\ \mathrm{H} & -3.02897900 & -0.48424600 \\ & -2.795000 & -1.48618300\end{array}$

Structure TS3-2to4-2

1 imaginary frequency $=1178.04 \mathrm{~cm}-1$

$\mathrm{E}($ b3lyp $)=-781.74591$ A.U.

Zero-point correction $=0.48205$ (Hartree/Particle)

Sum of electronic and zero-point Energies= -781.26386 A.U. 


\begin{tabular}{|c|c|c|c|}
\hline C & -0.76822000 & -1.20989000 & 1.31202200 \\
\hline C & -0.66542500 & -1.17413100 & -0.07975500 \\
\hline C & -1.74262300 & -0.39411800 & -0.90515500 \\
\hline C & -2.40077100 & 0.79853300 & -0.11850800 \\
\hline C & -2.83609000 & 0.34624300 & 1.29042200 \\
\hline C & -1.74646000 & -0.38271700 & 2.08907400 \\
\hline $\mathrm{H}$ & -3.18696700 & 1.20878000 & 1.86601800 \\
\hline $\mathrm{H}$ & -3.70022800 & -0.31847600 & 1.19930400 \\
\hline $\mathrm{H}$ & -1.16429300 & 0.30630600 & 2.71744200 \\
\hline $\mathrm{H}$ & -2.20324200 & -1.06500800 & 2.82198800 \\
\hline C & 0.10146900 & -2.08604000 & 2.16861300 \\
\hline $\mathrm{H}$ & 1.10074100 & -2.23733700 & 1.75737600 \\
\hline $\mathrm{H}$ & 0.19336500 & -1.67780300 & 3.17844200 \\
\hline $\mathrm{H}$ & -0.36219100 & -3.07796800 & 2.26389800 \\
\hline C & -1.66588000 & 2.17477000 & -0.11368500 \\
\hline C & 0.02595700 & -2.35580700 & -0.77993700 \\
\hline C & -0.34880300 & 2.19263500 & 0.60293500 \\
\hline $\mathrm{H}$ & -2.35959600 & 2.87513700 & 0.37355000 \\
\hline $\mathrm{H}$ & -1.56039900 & 2.53266600 & -1.14103700 \\
\hline C & 1.40040200 & -2.05033500 & -1.48438800 \\
\hline C & 2.29761800 & -1.30185700 & -0.55414000 \\
\hline $\mathrm{H}$ & 1.21341000 & -1.46411500 & -2.38689000 \\
\hline $\mathrm{H}$ & -3.32714200 & 1.00672900 & -0.66758600 \\
\hline C & -1.19557600 & 0.10629600 & -2.25792100 \\
\hline $\mathrm{H}$ & -0.86002300 & -0.72002900 & -2.89107300 \\
\hline $\mathrm{H}$ & -1.99398700 & 0.61681100 & -2.80580700 \\
\hline $\mathrm{H}$ & -0.37264500 & 0.81554300 & -2.15002500 \\
\hline C & -2.88177400 & -1.40993700 & -1.22911600 \\
\hline $\mathrm{H}$ & -3.70735700 & -0.88967600 & -1.72443200 \\
\hline $\mathrm{H}$ & -2.55047200 & -2.19701800 & -1.91099200 \\
\hline $\mathrm{H}$ & -3.27896200 & -1.89543200 & -0.33344000 \\
\hline $\mathrm{H}$ & 0.18284700 & -3.17267500 & -0.07365700 \\
\hline $\mathrm{H}$ & -0.63952500 & -2.74841600 & -1.54976900 \\
\hline $\mathrm{H}$ & 1.83902300 & -3.00486600 & -1.78678200 \\
\hline C & 1.99970500 & 0.03213900 & -0.34708500 \\
\hline $\mathrm{H}$ & 1.44255900 & 0.49974200 & -1.15814600 \\
\hline C & 2.83555000 & 1.01950900 & 0.45078800 \\
\hline $\mathrm{H}$ & 3.37458900 & 0.52659600 & 1.26478500 \\
\hline $\mathrm{H}$ & 3.60802300 & 1.37797300 & -0.24311700 \\
\hline C & 2.06002300 & 2.24515200 & 1.02587100 \\
\hline $\mathrm{H}$ & 1.72470000 & 2.00685900 & 2.04064300 \\
\hline $\mathrm{H}$ & 2.76214900 & 3.08217700 & 1.11399500 \\
\hline C & 0.85770800 & 2.60752100 & 0.18027400 \\
\hline $\mathrm{H}$ & 0.60851800 & -0.39649000 & 0.14951600 \\
\hline $\mathrm{H}$ & -0.37347300 & 1.78340500 & 1.61023000 \\
\hline
\end{tabular}




$\begin{array}{lrrr}\mathrm{C} & 3.35895900 & -2.06655100 & 0.17441500 \\ \mathrm{H} & 3.03666500 & -3.08481700 & 0.41655700 \\ \mathrm{H} & 4.22142100 & -2.17330300 & -0.50022300 \\ \mathrm{H} & 3.71584100 & -1.56994800 & 1.07783200 \\ \mathrm{C} & 1.13611200 & 3.32136900 & -1.11833600 \\ \mathrm{H} & 1.55687600 & 4.31325700 & -0.91043400 \\ \mathrm{H} & 1.88512500 & 2.79695300 & -1.72702400 \\ \mathrm{H} & 0.24336900 & 3.46773200 & -1.72947900 \\ * * * * * * * * * * * * * * * * * * * * * * * * * * * * * * * * * * * * * * * * * * * * * * * * * * * * * * * * * *\end{array}$

Structure TS3-2to6-2

1 imaginary frequency $=1203.01 \mathrm{~cm}-1$

$\mathrm{E}(\mathrm{b} 3 \mathrm{yp})=-781.74809 \mathrm{~A} . \mathrm{U}$.

Zero-point correction $=0.48158$ (Hartree/Particle)

Sum of electronic and zero-point Energies=-781.26651 A.U.

$\begin{array}{lrrr}\mathrm{C} & -1.25364400 & -1.08623900 & 1.23008600 \\ \mathrm{C} & -0.93231200 & -0.92897300 & -0.12398400 \\ \mathrm{C} & -1.87590700 & -0.04788400 & -1.00691200 \\ \mathrm{C} & -2.34521900 & 1.19207300 & -0.18353100 \\ \mathrm{C} & -3.07220900 & 0.75167400 & 1.09718600 \\ \mathrm{C} & -2.22858300 & -0.20407100 & 1.95022700 \\ \mathrm{H} & -3.34471400 & 1.62849400 & 1.69353400 \\ \mathrm{H} & -4.01605600 & 0.26704700 & 0.83417600 \\ \mathrm{H} & -1.64956300 & 0.34743100 & 2.70993300 \\ \mathrm{H} & -2.86674500 & -0.86363200 & 2.55629000 \\ \mathrm{C} & -0.59109200 & -2.09974900 & 2.11887500 \\ \mathrm{H} & 0.46682200 & -2.23575200 & 1.89141700 \\ \mathrm{H} & -0.69504500 & -1.82894200 & 3.17244800 \\ \mathrm{H} & -1.07807800 & -3.07657600 & 1.98667900 \\ \mathrm{C} & -1.20988300 & 2.21412100 & 0.10205900 \\ \mathrm{C} & -0.19340600 & -2.09685200 & -0.82237600 \\ \mathrm{C} & 0.06658000 & 1.64105600 & 0.70926300 \\ \mathrm{H} & -1.60776700 & 2.97802600 & 0.78324800 \\ \mathrm{H} & -0.97072600 & 2.73864700 & -0.82494400 \\ \mathrm{C} & 1.19808200 & -1.91831900 & -1.49310000 \\ \mathrm{C} & 2.28756900 & -1.44620800 & -0.55335600 \\ \mathrm{H} & 1.11888000 & -1.22427100 & -2.33340000 \\ \mathrm{H} & -3.07481300 & 1.72554400 & -0.80472200 \\ \mathrm{C} & -1.17545100 & 0.41176100 & -2.30392900 \\ \mathrm{H} & -0.98220400 & -0.43089400 & -2.97234000 \\ \mathrm{H} & -1.82542300 & 1.10779800 & -2.84449300 \\ \mathrm{H} & -0.22212800 & 0.91312200 & -2.12271000 \\ \mathrm{C} & -3.10621300 & -0.89457100 & -1.43058200 \\ \mathrm{H} & -3.82813900 & -0.26140900 & -1.95733700 \\ \mathrm{H} & -2.82027100 & -1.69523700 & -2.11791800\end{array}$




$\begin{array}{lrrr}\mathrm{H} & -3.61646100 & -1.35653500 & -0.58195200 \\ \mathrm{H} & -0.11826700 & -2.92904700 & -0.12024400 \\ \mathrm{H} & -0.87237200 & -2.45315700 & -1.60474400 \\ \mathrm{H} & 1.45375100 & -2.89733000 & -1.91889200 \\ \mathrm{C} & 2.65891300 & -0.15923900 & -0.63374800 \\ \mathrm{H} & 2.19939400 & 0.41339800 & -1.43942200 \\ \mathrm{C} & 3.48477000 & 0.67885400 & 0.29567500 \\ \mathrm{H} & 4.10672200 & 0.08878400 & 0.97324900 \\ \mathrm{H} & 4.16241600 & 1.32250000 & -0.27575300 \\ \mathrm{C} & 2.52866600 & 1.57287500 & 1.16831600 \\ \mathrm{H} & 2.18448800 & 0.99304500 & 2.02994500 \\ \mathrm{H} & 3.09635900 & 2.43258100 & 1.54988700 \\ \mathrm{C} & 1.34534900 & 2.06733400 & 0.38323100 \\ \mathrm{H} & -0.07841900 & 0.20688800 & 0.32782900 \\ \mathrm{H} & -0.00915900 & 1.30267200 & 1.74584200 \\ \mathrm{C} & 2.88444400 & -2.46332400 & 0.38813700 \\ \mathrm{H} & 2.12509600 & -3.09517600 & 0.86206100 \\ \mathrm{H} & 3.53325900 & -3.14568100 & -0.17628600 \\ \mathrm{H} & 3.49139600 & -2.01359900 & 1.17715700 \\ \mathrm{C} & 1.63372900 & 3.01173600 & -0.74573800 \\ \mathrm{H} & 1.45971900 & 4.03761500 & -0.38864200 \\ \mathrm{H} & 2.67304200 & 2.95839300 & -1.07527700 \\ \mathrm{H} & 0.97990200 & 2.86893000 & -1.60941300 \\ * * * * * * * * * * * * * * * * * * * * * * * * * * * * * * * * * * * * * * * * * * * * * * * * * * * * * * *\end{array}$

Structure TS4-2to6-2

1 imaginary frequency $=1023.96 \mathrm{~cm}-1$

$\mathrm{E}$ (b3lyp) $=-781.75168 \mathrm{~A} . \mathrm{U}$.

Zero-point correction $=0.481890$ (Hartree/Particle)

Sum of electronic and zero-point Energies= -781.269786 A.U.

$\begin{array}{lrrr}\mathrm{C} & 1.64811400 & 0.66439000 & 1.26539900 \\ \mathrm{C} & 1.56081600 & 0.69208300 & -0.08815600 \\ \mathrm{C} & 1.84956100 & -0.57957700 & -0.92719300 \\ \mathrm{C} & 1.47757000 & -1.88181800 & -0.14848400 \\ \mathrm{C} & 2.12109000 & -1.88464700 & 1.24865900 \\ \mathrm{C} & 1.82926700 & -0.61670800 & 2.06051800 \\ \mathrm{H} & 1.79828900 & -2.76994200 & 1.80877100 \\ \mathrm{H} & 3.20300700 & -1.99038400 & 1.12994000 \\ \mathrm{H} & 0.92931900 & -0.75379600 & 2.68252300 \\ \mathrm{H} & 2.63192100 & -0.46209500 & 2.79329200 \\ \mathrm{C} & 1.58680200 & 1.86335800 & 2.18891100 \\ \mathrm{H} & 1.49686400 & 2.82796800 & 1.69165700 \\ \mathrm{H} & 0.75215300 & 1.77157300 & 2.89709800 \\ \mathrm{H} & 2.49657700 & 1.90053200 & 2.80035800 \\ \mathrm{C} & -0.02637100 & -2.27636200 & -0.13004800\end{array}$




$\begin{array}{lrrr}\mathrm{C} & 1.38754600 & 2.00546900 & -0.84534200 \\ \mathrm{C} & -1.04425400 & -1.32394900 & 0.47863800 \\ \mathrm{H} & -0.08928400 & -3.21877400 & 0.43219400 \\ \mathrm{H} & -0.33740100 & -2.52963000 & -1.14854200 \\ \mathrm{C} & -0.02244900 & 2.29752900 & -1.47710100 \\ \mathrm{C} & -1.19176400 & 2.03175300 & -0.57835500 \\ \mathrm{H} & -0.14148400 & 1.73827000 & -2.40542900 \\ \mathrm{H} & 1.94383200 & -2.70450300 & -0.70714100 \\ \mathrm{C} & 1.15380600 & -0.60229900 & -2.30829300 \\ \mathrm{H} & 1.49701500 & 0.21383300 & -2.94979300 \\ \mathrm{H} & 1.40635800 & -1.53429100 & -2.82565600 \\ \mathrm{H} & 0.06396700 & -0.55088000 & -2.25092400 \\ \mathrm{C} & 3.37782500 & -0.58728500 & -1.22638000 \\ \mathrm{H} & 3.65174500 & -1.47201600 & -1.81261000 \\ \mathrm{H} & 3.66312800 & 0.29299900 & -1.81126200 \\ \mathrm{H} & 3.97722200 & -0.57771500 & -0.31429700 \\ \mathrm{H} & 1.65359200 & 2.84479600 & -0.20351700 \\ \mathrm{H} & 2.09229300 & 2.05062500 & -1.68307900 \\ \mathrm{H} & -0.03727500 & 3.36515400 & -1.73882700 \\ \mathrm{C} & -2.05134700 & 0.97590400 & -0.85588000 \\ \mathrm{H} & -1.97562800 & 0.58474500 & -1.87257600 \\ \mathrm{C} & -3.37502600 & 0.71908100 & -0.15108600 \\ \mathrm{H} & -3.76410000 & 1.62285600 & 0.32378800 \\ \mathrm{H} & -4.11310600 & 0.41266800 & -0.89730800 \\ \mathrm{C} & -3.27466800 & -0.40287100 & 0.96343800 \\ \mathrm{H} & -2.83088500 & 0.03288200 & 1.86391400 \\ \mathrm{H} & -4.28352800 & -0.74347300 & 1.20898900 \\ \mathrm{C} & -2.41949400 & -1.50559700 & 0.42960000 \\ \mathrm{H} & -1.31146400 & -0.10670700 & -0.31938300 \\ \mathrm{H} & -0.68722700 & -0.68269200 & 1.28259600 \\ \mathrm{C} & -1.39402400 & 2.92540700 & 0.60336000 \\ \mathrm{H} & -0.48225300 & 3.44597400 & 0.89397800 \\ \mathrm{H} & -2.12902600 & 3.69416700 & 0.32091200 \\ \mathrm{H} & -1.80366900 & 2.40202500 & 1.47129400 \\ \mathrm{C} & -3.09262600 & -2.58854100 & -0.35056800 \\ \mathrm{H} & -3.51127000 & -3.31045400 & 0.36644300 \\ \mathrm{H} & -34252100 & -2.20786000 & -0.92784600 \\ & -3.13777500 & -1.00937700\end{array}$

